\title{
Comparing Different Estimators of Parameters and Reliability for Mixed Weibull by Simulation
}

\author{
Dhwyia S. Hassun ${ }^{1}$, Nathier A, Ibrahim ${ }^{2}$, Assel N. Hussein ${ }^{3}$ \\ ${ }^{1}$ Proffessor \& Head, Department of Industrial Management, College of Administration \& \\ Economic, University of Baghdad, Iraq \\ ${ }^{2}$ Department of Banks Economics, College of Business Economics, Al - Nahrain University, \\ Iraq \\ ${ }^{3}$ College of Administration \& Economic, Iraqi University, Baghdad, Iraq
}

\begin{abstract}
This paper concerned with estimating reliability function of one of the important failure models, which is used when the underlying population is non homogenous, this model is called mixed Weibull distribution, when there are only two sub - population with mixing proportions taking into account the case when items that fail can classified and can be attributed to appropriate sub populations. Two methods were used to estimate the reliability function which are; (1) Maximum likelihood Method (ML). (2) Weighted Least Square Method (WLS). The simulation procedure using Monte Carlo Method, are used and several experiments are implemented to find the best estimators which have smallest mean square error. All results are explained in tables.
\end{abstract}

Keywords: Mixed Weibull Distribution, ML, WLS, Monte Carlo Method, SMSE, Scale parameters, Shape parameters

\section{INTRODUCTION}

Technological developments have witnessed the industrial sector in the last century, to the rapid development of industrial process and the emergence of electronic devices and systems in various areas of complex life such as medicine, the field of communications, space research, and military operations etc. The imposition of this development approach many researchers to configure constant and private non - hybrid (such as Weibull distribution, Gamma distribution, exponential distribution, and normal distribution), constantly technological advances received distributions mixed wide interest to prominence in many fields, including the field of reliability, the times are associated with failure assessment with reliability function mixture from two distributions or more, this mixture may due to the same distribution or two different distributions or more.

Because of mixed Weibull distribution is one of failure models by looking at the performance of the work equipment identical and independent as well as of great importance in the field of reliability increased attention and research to assess the optimization system reliability for this distribution statement operational life of a number of devices and equipment through their representation in one function to see how efficient this equipment and its ability to work for long periods of time, and therefore assess these machines and equipment for planning and development in the future in order to achieve the goal of developing production in terms of the longevity of both hardware and the lowest possible cost.

This distribution also called (Biweibull) ${ }^{[2]}$ and it appear from mixing two subpopulations each model is failure model, and is different from the second sum failure model. The (Biweibull) model is used for analysis of (Atmospheric Data) and (Aerospace scientists) and in stochastic process and its various applications such as Geometry, mathematics, theory of probability, and statistics.

The two subpopulation are represented in one function (1) as;

$f(t)=\alpha f_{1}(t)+(1-\alpha) f_{2}(t) \quad 0<\alpha<1$

where; 
$f_{1}(t)=\frac{p_{1}}{\theta_{1}} t^{p_{1}-1} e^{-\frac{t^{p_{1}}}{\theta_{1}}} t \geq 0, \theta_{1}, p_{1}>0$

$f_{2}(t)=\frac{p_{2}}{\theta_{2}} t^{p_{2}-1} e^{-} \frac{t^{p_{2}}}{\theta_{2}} t \geq 0, \theta_{2}, p_{2}>0$

$\left(\theta_{1}, \theta_{2}\right)$ are scales parameters

$\left(p_{1}, p_{2}\right)$ are shape parameters

$\left(\alpha_{s} \frac{n_{1}}{n}\right)$ mixing proportion parameter

While the cumulative distribution function $(C \cdot D . F)$ is written as;

$$
\begin{aligned}
& F(t)=\alpha F_{1}(t)+(1-\alpha) F_{2}(t) \\
& F(t)=1-\alpha e^{-\frac{t^{D_{1}}}{\theta_{1}}}-(1-\alpha) e^{-\frac{t^{D_{2}}}{\theta_{2}}}
\end{aligned}
$$

And the reliability function is;

$$
\begin{aligned}
& R(t)=\alpha R_{1}(t)+(1-\alpha) R_{2}(t) \\
& R(t)=\alpha e^{-\frac{t^{D_{1}}}{\theta_{1}}}-(1-\alpha) e^{-\frac{t^{D_{2}}}{\theta_{2}}}
\end{aligned}
$$

Method of estimating parameter2.1- Maximum likelihood method $(M L)$ :It is the important method to find the estimator of mixed weibull which consist of two subpopulation $\left(S p_{1}, S p_{2}\right)$ and parameter of (mixing proportion parameter). If $\left(t_{i j}\right)$ represents the time of failure of random sample $(n)$ taken randomly from Biweibull distribution, and we know when each unit belong to $\left(S p_{\mathrm{i}}, i=1,2\right)$, then we can determine the random variable $T$ (time) of failure of $\left(r_{\mathrm{i}}\right)$ units before (T) i.e $\left(t_{\mathrm{i} 1}, t_{\mathrm{i} 2}, \ldots, t_{\mathrm{i} v_{i}}\right)$, then the data of sample here is called time censored sampling (Type I), and the conditional probability distribution for time failure from $\left(S p_{\mathrm{i}}\right)$ before time $\mathrm{T}$ is;

$f_{i}(t \mid t \leq T)=\frac{f_{i}(t)}{F_{i}(T)} \quad 0 \leq t \leq T$

The probability that $\left(r_{1}\right)$ units from $\left(S p_{1}\right)$ failed before time $T$, and $\left(r_{2}\right)$ units from $\left(S p_{2}\right)$ failed before time $T$, and $(n-r)$, where $r=r_{1}+r_{2}$ is number of units survived till time $T$, this probability is defined through:

$\frac{n !}{r_{1} ! r_{2} !(n-\eta) !}\left[\alpha F_{1}(T)\right]^{r_{1}}\left[\beta F_{2}(T)\right]^{r_{2}}[R(T)]^{(n-r)}$
Then the likelihood function from this sample is; $L=\frac{n !}{r_{1} ! r_{2} !(n-r) !}\left[\alpha^{r_{1}} \beta^{r_{2}}\right]\left[\alpha e^{-\frac{T^{\eta_{1}}}{\theta_{1}}}+\beta e^{-\frac{T^{\eta_{2}}}{\theta_{2}}}\right]^{[(n-r)}$

$\left[\prod_{j=1}^{\gamma_{1}} \frac{p_{1}}{\theta_{1}}\left(T x_{1 j}\right)^{p_{1}-1} e^{-} \frac{\left(T x_{1 j}\right)^{p_{1}}}{\theta_{1}}\right]\left[\prod_{j=1}^{\gamma_{2}} \frac{p_{2}}{\theta_{2}}\left(T x_{i j}\right)^{p_{2}-1} e^{-\frac{\left(T x_{2 j}\right)^{p_{2}}}{\theta_{2}}}\right]$

Taking logarithm of both sides;

$\ln L=C+(n-r) \ln \left[\alpha e^{-} \frac{T^{p_{1}}}{\theta_{1}}+\beta e^{-} \frac{T^{p_{2}}}{\theta_{2}}\right]$

$+r_{1} \ln \alpha+r_{2} \ln \beta+\sum_{j=1}^{r_{1}}\left[\ln p_{1}-\ln \theta_{1}+\left(p_{1}-1\right) \ln \left(T x_{1 j}\right)-\frac{\left(T x_{1 j}\right)^{p_{1}}}{\theta_{1}}\right]$

$+\sum_{j=1}^{\gamma_{2}}\left[\ln p_{2}-\ln \theta_{2}+\left(p_{2}-1\right) \ln \left(T x_{2 j}\right)-\frac{\left(T x_{2 j}\right)^{p_{2}}}{\theta_{2}}\right]$

Now let;

$k=\frac{\alpha \theta^{-\frac{T D_{1}}{\theta_{1}}}}{\omega_{\theta}-\frac{T D_{1}}{\theta_{1}}+\beta_{\theta}-\frac{T D_{2}}{\theta_{2}}}$

Then from;

$\frac{\partial \ln L}{\partial \theta_{1}}=\frac{(n-r) \hat{k} T^{\hat{p}_{1}}}{\hat{\theta}_{1}^{2}}-\frac{r_{1}}{\hat{\theta}_{1}}+\frac{\sum_{j=1}^{\gamma_{1}}\left(T x_{1 j}\right)^{\hat{p}_{1}}}{\hat{\theta}_{1}^{2}}=0$
$\hat{\theta}_{1 M L E}=\frac{(n-n) \hat{k} T \hat{\eta}_{1} \sum_{j=1}^{r_{1}}\left(T x_{1 j}\right)^{\hat{\vartheta}_{1}}}{r_{1}}$

Also we can write $k$ as;

$k=\frac{1}{1+\frac{g}{\pi} e^{\left[\frac{T_{1}}{\theta_{1}}-\frac{T^{D 2}}{\theta_{2}}\right]}}$

Also;

$$
\begin{aligned}
& 1-k=\frac{\beta \theta^{-\frac{T D_{2}}{\theta_{2}}}}{\alpha \theta^{-\frac{T V_{1}}{\theta_{1}}+\beta \theta^{-}-\frac{T D_{2}}{\theta_{2}}}} \\
& 1-k=\frac{1}{1+\frac{\alpha}{\beta} e^{\left[-\frac{T V_{1}}{\theta_{1}}+\frac{T V_{2}}{\theta_{2}}\right]}}
\end{aligned}
$$


And;

$\frac{\partial \ln L}{\partial \theta_{2}}=\frac{(n-r)(1-\hat{k}) T^{\hat{p}_{2}}}{\hat{\theta}_{2}^{2}}-\frac{r_{2}}{\hat{\theta}_{2}}+\frac{\sum_{j=1}^{\gamma_{2}}\left(T x_{2 j}\right)^{\hat{p}_{2}}}{\widehat{\theta}_{2}^{2}}=0$

$\hat{\theta}_{2 M L E}=\frac{\left(n-r_{1}(1-\hat{k}) T \hat{\theta}_{2}+\sum_{j=1}^{r_{2}}\left(T x_{2 j}\right)^{\hat{\theta}_{2}}\right.}{r_{2}}$

(15) Also fro

deriving $\ln L$ with respect to shape parameter $\left(p_{1}\right)$, $\left(p_{2}\right)$ we found;

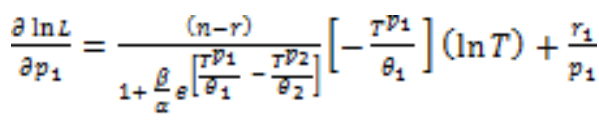

$+\sum_{j=1}^{\gamma_{1}} \ln \left(T x_{1 j}\right)\left(1-\frac{\left(T x_{1 j}\right)^{p_{11}}}{\theta_{1}}\right)$

Now let;

$g_{1}\left(\hat{p}_{1}\right)=\frac{-(n-r) k T^{D_{1}}(\ln T)}{\widehat{\theta}_{1}}+\frac{\hat{p}_{1}}{\hat{p}_{1}}+\sum_{j=1}^{\gamma_{1}}\left(T x_{1 j}\right)^{\hat{p}_{1}}(1-$ $\left.\frac{\left(T x_{1 j}\right)^{p / 1}}{\theta_{1}}\right)=0$

Also;

$$
\begin{aligned}
& \frac{\partial \ln L}{\partial p_{2}}=\frac{(n-F)}{1+\frac{\alpha}{g} \theta\left[-\frac{T V_{1}}{\theta_{1}}+\frac{T V_{2}}{\theta_{2}}\right]}\left[-\frac{T^{D_{2}}}{\theta_{2}}\right](\ln T)+\frac{p_{2}}{p_{2}} \\
& +\sum_{j=1}^{\gamma_{2}} \ln \left(T x_{2 j}\right)\left(1-\frac{\left(T x_{2 j}\right)^{D_{2}}}{\theta_{2}}\right)
\end{aligned}
$$

Also suppose;

$$
\begin{aligned}
& g_{2}\left(\hat{p}_{2}\right)=\frac{-(n-r)(1-\hat{K}) r^{\hat{\eta}}(\ln T)}{\ddot{\theta}_{2}}+\frac{r_{2}}{\hat{p}_{2}} \\
& +\sum_{j=1}^{\gamma_{2}}\left(T x_{2 j}\right)^{\hat{\gamma}_{2}}\left(1-\frac{\left(T x_{2 j}\right)^{D_{2}}}{\widehat{\theta}_{2}}\right)=0
\end{aligned}
$$

Similarly;

$\frac{\partial \ln L}{\partial \alpha}=\frac{r_{1}}{\alpha}-\frac{r_{2}}{\beta}+\frac{(n-r)\left[\exp \left(-\frac{T^{p_{1}}}{\theta_{1}}\right)-\exp \left(-\frac{T^{p_{2}}}{\theta_{2}}\right)\right]}{\alpha \exp \left(-\frac{T^{p_{1}}}{\theta_{1}}\right)+\beta \exp \left(-\frac{T^{p_{2}}}{\theta_{2}}\right)}$

After simplification, we obtain,

$$
\frac{\partial \ln L}{\partial \alpha}=\frac{k(n-r)+r_{1}}{\alpha}-\frac{(1-k)(n-r)+r_{2}}{\beta}
$$

$\therefore \beta=1-\alpha$ $\frac{\partial \ln L}{\partial \alpha}=\frac{k(n-r)+r_{1}-\alpha r_{1}-\alpha(n-r)-\alpha r_{2}}{\alpha(1-\alpha)}$

$\hat{\alpha}=\left[\hat{k}(n-r)+r_{1}\right] /\left(r_{1}+n-r+r_{2}\right)$

Therefore;

$\hat{\alpha}_{M L E}=\hat{k}\left(\frac{n-r}{n}\right)+\frac{r_{1}}{n}$

Finally, rewrite equations $(12,13,15,18)$;

$$
\begin{aligned}
& \hat{\lambda_{1}}=\frac{(n-r) \hat{k}}{r_{1}}+\bar{y}_{1} \\
& \hat{\lambda}_{2}=\frac{(n-r)(1-\hat{k})}{r_{2}}+\bar{y}_{2} \\
& \hat{\alpha}=\frac{(n-r) \hat{k}+r_{1}}{n} \\
& \hat{k}=\frac{1}{1+\frac{\hat{\beta}}{\hat{\alpha}} \exp \left[\frac{1}{\hat{\lambda}_{1}}-\frac{1}{\hat{\lambda}_{2}}\right]}
\end{aligned}
$$

Such that;

$$
\hat{\lambda}_{i}=\frac{\hat{\theta}_{i}}{T \hat{p}_{i}} \quad \bar{y}_{i}=\frac{\sum_{j=1}^{r_{i}}\left(x_{i j}\right)^{\bar{p}_{i}}}{r_{i}}
$$

To find maximum likelihood estimator for $p_{1}, p_{2}$ substitute the proposed values for $\left(\alpha^{*}, p_{1}^{*}, p_{2}^{*}, \theta_{1}^{*}, \theta_{2}^{*}\right)$ in the equation;

$$
k^{*}=\frac{1}{1+\frac{Q^{*}}{\alpha^{*}}\left[\frac{T D_{1}^{*}}{\theta_{1}^{*}}-\frac{T D_{2}^{*}}{\theta_{2}^{*}}\right]}
$$

Applying direct search technique such that;

$$
g\left(\hat{k}_{0}\right)-\hat{k}_{0}=0 \quad 0 \leq k \leq 1
$$

We can find the two parameters, $p_{01}, p_{02}$ from equations (17 \&18). This is explained through simulation procedure.

Since the estimator's of maximum likelihood method are invariant, so we can use, $\left(\hat{\theta}_{1 M L}, \hat{\theta}_{2 M L}, \hat{\alpha}_{M L}, \hat{p}_{1 M L}, \hat{p}_{2 M L}\right)$ to find the maximum likelihood estimator of reliability function for Biweibull, from equation; 
$\hat{R}_{M L}(t)=\hat{\alpha} \exp \left[-\left(\frac{t^{D_{1}}}{\hat{\theta}_{1}}\right)\right]+(1-\hat{\alpha}) \exp \left[-\left(\frac{t^{D_{2}}}{\hat{\theta}_{2}}\right)\right]$

\section{Weighted least Square method}

The aim of this method is to find the estimator's of parameters from minimizing the total sum squares of deviations, and it depend on (White's Method) and application it on reliability function as follows;

Suppose $\left(t_{i j}\right)$ are independent identically distributed random variable represent the time of failure and have;

$R_{i}(t)=\exp \left[-\left(\frac{t^{p_{i}}}{\theta_{i}}\right)\right]$

$t \in(0, \infty) \quad 0<\theta_{\mathrm{i}}<p_{\mathrm{i}}<\infty \quad \forall i=1,2 \quad$ (25)

Let $t_{\tilde{j}}=\frac{T}{M}$ failure time for each subpopulation for units that failed before time $T$, where $T$ is time through it, the time failure $\left(t_{j}\right)$ are determined i.e;

$0=t_{0}<t_{1}<t_{2}<\cdots<t_{m-1}<t_{m}=T$

$m$ : The total number of period through $(0, T)$.

$w_{i j^{m}}$ Number of observation for time to failure during $\left(t_{j-1}-t_{j}\right)$ for each subpopulation, $i=1,2 \quad j=-1,2, \ldots, m$.

$\alpha=\frac{n_{1}}{n}$ mixing parameter

$\theta_{i}:$ Scale parameter

$p_{\mathrm{i}}:$ Shape parameter

Taking two times logarithm for equation (25) we get;

$\ln \ln \left[R_{\mathrm{i}}(t)\right]^{-1}=p_{\mathrm{i}} \ln t_{\mathrm{i}}-\ln \theta_{\mathrm{i}}$

Let;

$y_{\mathrm{i} j}=\ln \ln \left[n_{\mathrm{i}} /\left(n_{\mathrm{i}}-\Sigma_{k=1}^{j} w_{i k}\right)\right]$

And;

$A_{\mathrm{i}}=-\ln \theta_{\mathrm{i}} \quad z_{\mathrm{i}}=\ln t_{\mathrm{i}} \quad \forall i=1,2 \quad j=1,2, \ldots, m$
Using equation of simple regression;

$y_{i j}=A_{\mathrm{i}}+p_{\mathrm{i}} z_{j}+\epsilon_{\mathrm{i}}$

Then the weighted least square estimators of parameters $\left(n_{\mathrm{i}}, \theta_{\mathrm{i}}, p_{\mathrm{i}}, i=1,2\right)$ are obtained from;

$Q\left(n_{\mathrm{i}}, \theta_{\mathrm{i}}, p_{\mathrm{i}}, \quad i=1,2\right)=\sum_{\mathrm{i}=1}^{2} \sum_{j=1}^{m} w_{\mathrm{i} j}\left(Y_{\mathrm{i} j}-A_{\mathrm{i}}-p_{\mathrm{i}} z_{j}\right)^{2}$

Chengel Fu. (1982) proposed that the sub sample size $\left(n_{1}, n_{2}\right)$ are unknown, and $n_{1}$ is one of $(k=1)$ of possible values;

$n_{1}^{*}=\left\{\sum_{j=1}^{m} w_{1 j}, \sum_{j=1}^{m} w_{1 j}+1, \ldots, n-\sum_{j=1}^{m} w_{2 j}\right\}$

And;

$k=n-\sum_{i=1}^{2} \sum_{j=1}^{m} w_{i j}$

$$
n_{2}=n-n_{1}^{*}
$$

Therefore, the weighted least squares estimators for $A_{\mathrm{i}}, p_{\mathrm{i}}$ are;

$\hat{p}_{\mathrm{i}}=\left[\sum_{j=1}^{m} w_{i j} z_{j j} y_{i j}-\left(\sum_{j=1}^{m} w_{i j} z_{j}\right)\left(\sum_{j=1}^{m} w_{i j} y_{i j}\right) / w_{i j}\right] / D_{i}$

$D_{\mathrm{i}}=\left[\sum_{j=1}^{\mathrm{m}} w_{\mathrm{i} j} z_{j}^{2}-\left(\sum_{j=1}^{\mathrm{m}} w_{\mathrm{ij}} z_{j}\right)^{2} / w_{\mathrm{i}}\right]$

$\hat{A}_{i}=\left(\sum_{j=1}^{m} w_{i j} y_{i j}\right)-\hat{p}_{i}\left(\sum_{j=1}^{m} w_{i j} z_{j j}\right) / w_{i}$

$$
w_{i}=\sum_{j=1}^{m} w_{i j} \quad j=1,2
$$

The weighted least square's estimator's for parameter's $\left(A_{\mathrm{i}}, p_{\mathrm{i}}, n_{\mathrm{i}}\right)$ are;

$\AA_{\mathrm{i}}, \tilde{p}_{\mathrm{i}}, \tilde{n}_{\mathrm{i}}$ which satisfy equation (34);

$Q\left(A_{i}, \tilde{p}_{i}, \tilde{n}_{i}, i=1,2\right)=\min _{n_{1} \in S}\left\{Q\left(A_{i}, \tilde{p}_{\mathrm{i}}, \tilde{n}_{i}, i=\right.\right.$ $1,2)\}$

So; 
$\hat{\alpha}=\frac{\mathfrak{m}_{1}}{n}$

$$
\theta_{i}=\exp \left(-A_{i}\right)
$$

The estimators $\left(\mathscr{\theta}_{i}, \hat{a}\right)$ are function of $w_{i j}$ depend on (time controlling $\mathrm{T}$ ), and number of time period $m$.

Finally we can find approximate estimator of reliability function;

$$
\begin{aligned}
& \hat{R}_{W L s}(t)=\alpha \hat{R}_{1}(t)+(1-\alpha) \hat{R}_{2}(t) \\
& \hat{R}_{W L s}(t)=\hat{\alpha} \exp \left[-\left(\frac{t^{\hat{p}_{1}}}{\hat{\theta}_{1}}\right)\right]+(1-\hat{\alpha}) \exp \left[-\left(\frac{t^{\hat{p}_{2}}}{\hat{\theta}_{2}}\right)\right]
\end{aligned}
$$

\section{Simulation Procedures}

To find the estimator's (ML\&WLS) we perform simulation experiments using Monte Carlo assuming that;

\begin{tabular}{|l|l|l|l|l|l|l|l|l|l|}
\hline$\alpha$ & \multicolumn{4}{|l|}{0.3} & \multicolumn{4}{l|}{0.4} & 0.5 \\
\hline$n$ & 15 & 20 & 25 & 15 & 20 & 25 & 15 & 20 & 25 \\
& 0 & 0 & 0 & 0 & 0 & 0 & 0 & 0 & 0 \\
\hline$n_{1}$ & 45 & 60 & 75 & 60 & 80 & $\begin{array}{l}10 \\
0\end{array}$ & 75 & $\begin{array}{l}10 \\
0\end{array}$ & $\begin{array}{l}12 \\
5\end{array}$ \\
\hline
\end{tabular}

Also the estimated values for $\left(p_{1}, p_{2}\right)$ and $\left(\theta_{1}, \theta_{2}\right)$ are given in the table;

\begin{tabular}{|l|l|l|l|l|}
\hline Model & $p_{1}$ & $\theta_{1}$ & $p_{2}$ & $\theta_{2}$ \\
\hline$I$ & 1 & 2 & 1 & 3 \\
\hline$I I$ & 0.8 & 3 & 0.9 & 4 \\
\hline$I I I$ & 0.7 & 5 & 1.2 & 3 \\
\hline
\end{tabular}

And also the chosen values for predetermined censoring time $(T)$ :

\begin{tabular}{|l|l|l|}
\hline Model & $\mathrm{T}$ \\
\hline$I$ & 2 & 4 \\
\hline$I I$ & 4 & 5 \\
\hline$I I I$ & 3 & 4 \\
\hline
\end{tabular}

Also the total number of time intervals $(m)$ are;

\begin{tabular}{|l|l|l|}
\hline Model & $\mathrm{m}$ \\
\hline$I$ & 4 & 8 \\
\hline$I I$ & 8 & 10 \\
\hline$I I I$ & 6 & 8 \\
\hline
\end{tabular}

And applying $t_{1 j}, t_{2 j}$ as follows.

$t_{1 j}=\theta_{1 j}\left(-\ln \left(1-u_{j}\right)\right)^{\frac{1}{p_{1}}} t_{2 j}=\theta_{2 j}\left(-\ln \left(1-u_{j}\right)\right)^{\frac{1}{p_{2}}} u_{j} \sim$ uniform $(0,1$

The comparison between estimators are done using (MSE) and the replication for each experiment is $(L=1000)$. To find the best estimator for reliability function, we use the integrated mean square error (IMSE) for comparison.

Where;

$\operatorname{IMSE}[\hat{R}(t)]=\frac{1}{L} \sum_{j=1}^{L}\left[\frac{1}{n_{t}} \sum_{i=1}^{n_{\mathrm{t}}} \hat{R_{j}}\left(t_{j}\right)-R\left(t_{i}\right)\right]^{2}$

$=\frac{1}{n_{t}} \sum_{i=1}^{n_{t}} \operatorname{MSE}\left[\hat{R}_{j}\left(t_{j}\right)\right] \quad i=1,2, \ldots, n_{t}$

$n_{t}$ is the limit of $t_{i}$ from lower bound to upper.

The results for the estimator's of parameter's are explained in tables $(1,2,3,4,12,13,14,15)$. Also the estimator's of reliability function are explained in tables $(5,6,7,8,9,10,11,16,17,18,19,20,21$, 22). 
Table (1): values of estimating mixed weibull distribution parameters for two partial population by MLE method for first model and frequency experiment $(L=1000)$.

\begin{tabular}{|c|c|c|c|c|c|c|c|c|}
\hline$\alpha$ & $n$ & $T$ & $M$ & $p_{1}$ & $p_{2}$ & $\theta_{1}$ & $\theta_{2}$ & $\alpha$ \\
\hline \multirow{6}{*}{0.3} & \multirow[t]{2}{*}{150} & 2 & 4 & 0.95917 & 0.97125 & 2.27668 & 2.82501 & 0.32832 \\
\hline & & 4 & 8 & 0.97979 & 0.98301 & 2.12388 & 2.87417 & 0.31059 \\
\hline & \multirow[t]{2}{*}{200} & 2 & 4 & 0.96529 & 0.97351 & 2.28925 & 2.83030 & 0.32770 \\
\hline & & 4 & 8 & 0.98518 & 0.98613 & 2.10654 & 2.89990 & 0.30865 \\
\hline & \multirow[t]{2}{*}{250} & 2 & 4 & 0.96981 & 0.97798 & 2.27112 & 2.83343 & 0.32559 \\
\hline & & 4 & 8 & 0.98632 & 0.98868 & 2.09976 & 2.90433 & 0.30742 \\
\hline \multirow{6}{*}{0.4} & \multirow[t]{2}{*}{150} & 2 & 4 & 0.96550 & 0.96837 & 2.14224 & 2.85109 & 0.42121 \\
\hline & & 4 & 8 & 0.98326 & 0.98179 & 2.07427 & 2.87003 & 0.40950 \\
\hline & \multirow[t]{2}{*}{200} & 2 & 4 & 0.97073 & 0.96971 & 2.15688 & 2.84987 & 0.41982 \\
\hline & & 4 & 8 & 0.98824 & 0.98507 & 2.08222 & 2.88288 & 0.40859 \\
\hline & \multirow[t]{2}{*}{250} & 2 & 4 & 0.97422 & 0.97718 & 2.12140 & 2.87480 & 0.41631 \\
\hline & & 4 & 8 & 0.98833 & 0.98763 & 2.04748 & 2.91000 & 0.40551 \\
\hline \multirow{6}{*}{0.5} & \multirow[t]{2}{*}{150} & 2 & 4 & 0.96897 & 0.96633 & 2.04163 & 2.91477 & 0.51053 \\
\hline & & 4 & 8 & 0.98489 & 0.97961 & 2.03312 & 2.87765 & 0.50740 \\
\hline & \multirow[t]{2}{*}{200} & 2 & 4 & 0.97628 & 0.96561 & 2.07463 & 2.88542 & 0.51334 \\
\hline & & 4 & 8 & 0.98954 & 0.98250 & 2.02680 & 2.91009 & 0.50497 \\
\hline & \multirow[t]{2}{*}{250} & 2 & 4 & 0.97685 & 0.97489 & 2.04874 & 2.91411 & 0.50881 \\
\hline & & 4 & 8 & 0.98909 & 0.98745 & 2.01314 & 2.92456 & 0.50278 \\
\hline
\end{tabular}

Table (2): values of estimating mixed weibull distribution parameters for two partial population by WLS method for first model and frequency experiment $(L=1000)$.

\begin{tabular}{|c|c|c|c|c|c|c|c|c|}
\hline$\alpha$ & $n$ & $T$ & $M$ & $p_{1}$ & $p_{2}$ & $\theta_{1}$ & $\theta_{2}$ & $\alpha$ \\
\hline \multirow{6}{*}{0.3} & \multirow[t]{2}{*}{150} & 2 & 4 & 1.08147 & 1.11702 & 2.70819 & 2.64834 & 0.38072 \\
\hline & & 4 & 8 & 1.00545 & 1.05907 & 2.38666 & 2.87317 & 0.33975 \\
\hline & \multirow[t]{2}{*}{200} & 2 & 4 & 1.06834 & 1.09248 & 2.72002 & 2.63603 & 0.37799 \\
\hline & & 4 & 8 & 1.00507 & 1.04359 & 2.36215 & 2.86114 & 0.33592 \\
\hline & \multirow[t]{2}{*}{250} & 2 & 4 & 1.05193 & 1.08582 & 2.71779 & 2.62152 & 0.37702 \\
\hline & & 4 & 8 & 1.00721 & 1.03636 & 2.30821 & 2.87719 & 0.32975 \\
\hline \multirow{6}{*}{0.4} & \multirow[t]{2}{*}{150} & 2 & 4 & 1.07541 & 1.12308 & 2.36727 & 2.74482 & 0.45679 \\
\hline & & 4 & 8 & 1.01144 & 1.06092 & 2.21510 & 2.91734 & 0.43048 \\
\hline & \multirow[t]{2}{*}{200} & 2 & 4 & 1.06357 & 1.09903 & 2.39866 & 2.70462 & 0.45575 \\
\hline & & 4 & 8 & 1.01214 & 1.04335 & 2.21811 & 2.88289 & 0.42725 \\
\hline & \multirow[t]{2}{*}{250} & 2 & 4 & 1.04208 & 1.09697 & 2.42001 & 2.66176 & 0.46076 \\
\hline & & 4 & 8 & 1.00910 & 1.03854 & 2.17323 & 2.91017 & 0.42240 \\
\hline \multirow{6}{*}{0.5} & \multirow[t]{2}{*}{150} & 2 & 4 & 1.07144 & 1.13225 & 2.17917 & 2.85809 & 0.53521 \\
\hline & & 4 & 8 & 1.01822 & 1.06442 & 2.11860 & 2.97230 & 0.52135 \\
\hline & \multirow[t]{2}{*}{200} & 2 & 4 & 1.05795 & 1.10727 & 2.21584 & 2.77786 & 0.53861 \\
\hline & & 4 & 8 & 1.01657 & 1.04480 & 2.12134 & 2.92983 & 0.51932 \\
\hline & \multirow[t]{2}{*}{250} & 2 & 4 & 1.04219 & 1.10122 & 2.20555 & 2.77828 & 0.53676 \\
\hline & & 4 & 8 & 1.00812 & 1.04236 & 2.10358 & 2.93806 & 0.51639 \\
\hline
\end{tabular}


Table (3): values of MSE of mixed weibull distribution parameters for two partial population by MLE method for first model and frequency experiment $(\mathrm{L}=1000)$.

\begin{tabular}{|c|c|c|c|c|c|c|c|c|}
\hline$\alpha$ & $n$ & $T$ & $M$ & $p_{1}$ & $p_{1}$ & $\theta_{1}$ & $\theta_{2}$ & $\alpha$ \\
\hline \multirow{6}{*}{0.3} & \multirow[t]{2}{*}{150} & 2 & 4 & 0.00841 & 0.00530 & 1.20549 & 0.48303 & 0.00973 \\
\hline & & 4 & 8 & 0.00307 & 0.00250 & 0.39994 & 0.17899 & 0.00155 \\
\hline & \multirow[t]{2}{*}{200} & 2 & 4 & 0.00660 & 0.00425 & 0.95582 & 0.37081 & 0.00705 \\
\hline & & 4 & 8 & 0.00225 & 0.00189 & 0.25089 & 0.12503 & 0.00093 \\
\hline & \multirow[t]{2}{*}{250} & 2 & 4 & 0.00517 & 0.00333 & 0.79017 & 0.29677 & 0.00554 \\
\hline & & 4 & 8 & 0.00186 & 0.00151 & 0.24503 & 0.11093 & 0.00082 \\
\hline \multirow{6}{*}{0.4} & \multirow[t]{2}{*}{150} & 2 & 4 & 0.00652 & 0.00599 & 0.56613 & 0.54431 & 0.00774 \\
\hline & & 4 & 8 & 0.00240 & 0.00288 & 0.23515 & 0.23303 & 0.00161 \\
\hline & \multirow[t]{2}{*}{200} & 2 & 4 & 0.00512 & 0.00502 & 0.50977 & 0.45663 & 0.00642 \\
\hline & & 4 & 8 & 0.00182 & 0.00209 & 0.18819 & 0.17098 & 0.00116 \\
\hline & \multirow[t]{2}{*}{250} & 2 & 4 & 0.00432 & 0.00361 & 0.38615 & 0.34306 & 0.00478 \\
\hline & & 4 & 8 & 0.00154 & 0.00169 & 0.15306 & 0.13892 & 0.00090 \\
\hline \multirow{6}{*}{0.5} & \multirow[t]{2}{*}{150} & 2 & 4 & 0.00543 & 0.00669 & 0.33296 & 0.66843 & 0.00681 \\
\hline & & 4 & 8 & 0.00207 & 0.00328 & 0.18398 & 0.30062 & 0.00167 \\
\hline & \multirow[t]{2}{*}{200} & 2 & 4 & 0.00392 & 0.00570 & 0.30346 & 0.57911 & 0.00597 \\
\hline & & 4 & 8 & 0.00153 & 0.00241 & 0.13574 & 0.21015 & 0.00108 \\
\hline & \multirow[t]{2}{*}{250} & 2 & 4 & 0.00360 & 0.00426 & 0.23884 & 0.44462 & 0.00453 \\
\hline & & 4 & 8 & 0.00136 & 0.00180 & 0.10944 & 0.17303 & 0.00085 \\
\hline
\end{tabular}

Table (4): values of MSE of mixed weibull distribution parameters for two partial population by WLS method for first model and frequency experiment $(\mathrm{L}=1000)$.

\begin{tabular}{|c|c|c|c|c|c|c|c|c|}
\hline$\alpha$ & $n$ & $T$ & $M$ & $p_{1}$ & $p_{1}$ & $\theta_{1}$ & $\theta_{2}$ & $\alpha$ \\
\hline \multirow{6}{*}{0.3} & \multirow[t]{2}{*}{150} & 2 & 4 & 0.12923 & 0.08318 & 2.92874 & 1.16049 & 0.03302 \\
\hline & & 4 & 8 & 0.05152 & 0.02995 & 0.84602 & 0.39375 & 0.00640 \\
\hline & \multirow[t]{2}{*}{200} & 2 & 4 & 0.10637 & 0.05844 & 2.83919 & 1.01496 & 0.03028 \\
\hline & & 4 & 8 & 0.04156 & 0.02048 & 0.66655 & 0.29481 & 0.00548 \\
\hline & \multirow[t]{2}{*}{250} & 2 & 4 & 0.08547 & 0.05162 & 2.64963 & 0.92778 & 0.02858 \\
\hline & & 4 & 8 & 0.03626 & 0.01699 & 0.55954 & 0.25075 & 0.00449 \\
\hline \multirow{6}{*}{0.4} & \multirow[t]{2}{*}{150} & 2 & 4 & 0.09761 & 0.09584 & 1.46758 & 1.41319 & 0.02814 \\
\hline & & 4 & 8 & 0.03820 & 0.03363 & 0.41187 & 0.49058 & 0.00536 \\
\hline & \multirow[t]{2}{*}{200} & 2 & 4 & 0.07892 & 0.07321 & 1.45420 & 1.22436 & 0.02618 \\
\hline & & 4 & 8 & 0.03148 & 0.02462 & 0.35211 & 0.33936 & 0.00456 \\
\hline & \multirow[t]{2}{*}{250} & 2 & 4 & 0.06171 & 0.06067 & 1.30284 & 1.08419 & 0.02476 \\
\hline & & 4 & 8 & 0.02736 & 0.01899 & 0.28066 & 0.29442 & 0.00372 \\
\hline \multirow{6}{*}{0.5} & \multirow[t]{2}{*}{150} & 2 & 4 & 0.07943 & 0.10924 & 0.87802 & 1.86142 & 0.02464 \\
\hline & & 4 & 8 & 0.03101 & 0.04045 & 0.26179 & 0.63660 & 0.00464 \\
\hline & \multirow[t]{2}{*}{200} & 2 & 4 & 0.06317 & 0.08790 & 0.84012 & 1.52901 & 0.02297 \\
\hline & & 4 & 8 & 0.02607 & 0.02964 & 0.21762 & 0.42236 & 0.00387 \\
\hline & \multirow[t]{2}{*}{250} & 2 & 4 & 0.04891 & 0.07161 & 0.74203 & 1.40899 & 0.02111 \\
\hline & & 4 & 8 & 0.02028 & 0.02311 & 0.17062 & 0.36562 & 0.00318 \\
\hline
\end{tabular}


Table (5): values of reliability function estimator \& MSE for estimating reliability function by all methods for first model when $(\alpha=0.3, T=2)$ for $L=1000$.

\begin{tabular}{|c|c|c|c|c|c|c|}
\hline$n$ & $t_{i}$ & Real & $M L E$ & WLS & $\begin{array}{l}M S E(\hat{R}) \\
M L E\end{array}$ & $\begin{array}{l}M S E(\hat{R}) \\
W L S\end{array}$ \\
\hline \multirow{11}{*}{150} & 0.5 & 0.82617 & 0.81650 & 0.82808 & 0.00040 & 0.00085 \\
\hline & 1.0 & 0.68353 & 0.67634 & 0.68038 & 0.00074 & 0.00107 \\
\hline & 1.5 & 0.56628 & 0.56382 & 0.56301 & 0.00111 & 0.00127 \\
\hline & 2.0 & 0.46975 & 0.47230 & 0.47298 & 0.00150 & 0.00157 \\
\hline & 2.5 & 0.39017 & 0.39725 & 0.40316 & 0.00186 & 0.00219 \\
\hline & 3.0 & 0.32445 & 0.33533 & 0.34759 & 0.00218 & 0.00317 \\
\hline & 3.5 & 0.27011 & 0.28396 & 0.30232 & 0.00245 & 0.00433 \\
\hline & 4.0 & 0.22511 & 0.24115 & 0.26483 & 0.00271 & 0.00548 \\
\hline & 4.5 & 0.18781 & 0.20532 & 0.23342 & 0.00298 & 0.00649 \\
\hline & 5.0 & 0.15683 & 0.17522 & 0.20687 & 0.00331 & 0.00729 \\
\hline & 5.5 & 0.13109 & 0.14983 & 0.18426 & 0.00376 & 0.00787 \\
\hline \multirow{11}{*}{200} & 0.5 & 0.82617 & 0.81832 & 0.82775 & 0.00031 & 0.00065 \\
\hline & 1.0 & 0.68353 & 0.67815 & 0.68113 & 0.00059 & 0.00084 \\
\hline & 1.5 & 0.56628 & 0.56527 & 0.56455 & 0.00091 & 0.00103 \\
\hline & 2.0 & 0.46975 & 0.47330 & 0.47432 & 0.00124 & 0.00132 \\
\hline & 2.5 & 0.39017 & 0.39782 & 0.40389 & 0.00153 & 0.00189 \\
\hline & 3.0 & 0.32445 & 0.33552 & 0.34775 & 0.00176 & 0.00274 \\
\hline & 3.5 & 0.27011 & 0.28387 & 0.30207 & 0.00191 & 0.00375 \\
\hline & 4.0 & 0.22511 & 0.24086 & 0.26430 & 0.00199 & 0.00475 \\
\hline & 4.5 & 0.18781 & 0.20493 & 0.23268 & 0.00200 & 0.00562 \\
\hline & 5.0 & 0.15683 & 0.17481 & 0.20595 & 0.00195 & 0.00632 \\
\hline & 5.5 & 0.13109 & 0.14948 & 0.18317 & 0.00187 & 0.00681 \\
\hline \multirow{11}{*}{250} & 0.5 & 0.82617 & 0.81943 & 0.82746 & 0.00025 & 0.00055 \\
\hline & 1.0 & 0.68353 & 0.67860 & 0.68101 & 0.00050 & 0.00074 \\
\hline & 1.5 & 0.56628 & 0.56475 & 0.56427 & 0.00078 & 0.00091 \\
\hline & 2.0 & 0.46975 & 0.47183 & 0.47348 & 0.00105 & 0.00110 \\
\hline & 2.5 & 0.39017 & 0.39551 & 0.40238 & 0.00128 & 0.00152 \\
\hline & 3.0 & 0.32445 & 0.33253 & 0.34566 & 0.00145 & 0.00221 \\
\hline & 3.5 & 0.27011 & 0.28034 & 0.29955 & 0.00155 & 0.00308 \\
\hline & 4.0 & 0.22511 & 0.23696 & 0.26146 & 0.00159 & 0.00398 \\
\hline & 4.5 & 0.18781 & 0.20077 & 0.22960 & 0.00158 & 0.00479 \\
\hline & 5.0 & 0.15683 & 0.17050 & 0.20270 & 0.00152 & 0.00544 \\
\hline & 5.5 & 0.13109 & 0.14511 & 0.17980 & 0.00144 & 0.00591 \\
\hline
\end{tabular}


Table (6): values of reliability function estimator \& MSE for estimating reliability function by all methods for first model when $(\alpha=0.3, T=4)$ for $L=1000$.

\begin{tabular}{|c|c|c|c|c|c|c|}
\hline$n$ & $t_{\mathrm{i}}$ & Real & $M L E$ & WLS & $\begin{array}{l}M S E(\hat{R}) \\
M L E\end{array}$ & $\begin{array}{l}\text { MSE }(\hat{R}) \\
\text { WLS }\end{array}$ \\
\hline \multirow{11}{*}{150} & 0.5 & 0.82617 & 0.81893 & 0.82670 & 0.00021 & 0.00077 \\
\hline & 1.0 & 0.68353 & 0.67599 & 0.68266 & 0.00044 & 0.00104 \\
\hline & 1.5 & 0.56628 & 0.56023 & 0.56302 & 0.00068 & 0.00110 \\
\hline & 2.0 & 0.46975 & 0.46578 & 0.46510 & 0.00088 & 0.00116 \\
\hline & 2.5 & 0.39017 & 0.38836 & 0.38560 & 0.00105 & 0.00121 \\
\hline & 3.0 & 0.32445 & 0.32464 & 0.32125 & 0.00116 & 0.00124 \\
\hline & 3.5 & 0.27011 & 0.27204 & 0.26911 & 0.00121 & 0.00126 \\
\hline & 4.0 & 0.22511 & 0.22848 & 0.22674 & 0.00121 & 0.00127 \\
\hline & 4.5 & 0.18781 & 0.19231 & 0.19211 & 0.00118 & 0.00127 \\
\hline & 5.0 & 0.15683 & 0.16221 & 0.16367 & 0.00112 & 0.00128 \\
\hline & 5.5 & 0.13109 & 0.13710 & 0.14016 & 0.00104 & 0.00128 \\
\hline \multirow{11}{*}{200} & 0.5 & 0.82617 & 0.82101 & 0.82679 & 0.00015 & 0.00059 \\
\hline & 1.0 & 0.68353 & 0.67829 & 0.68317 & 0.00034 & 0.00082 \\
\hline & 1.5 & 0.56628 & 0.56219 & 0.56420 & 0.00052 & 0.00089 \\
\hline & 2.0 & 0.46975 & 0.46720 & 0.46673 & 0.00068 & 0.00093 \\
\hline & 2.5 & 0.39017 & 0.38917 & 0.38732 & 0.00081 & 0.00096 \\
\hline & 3.0 & 0.32445 & 0.32487 & 0.32275 & 0.00088 & 0.00097 \\
\hline & 3.5 & 0.27011 & 0.27174 & 0.27019 & 0.00091 & 0.00098 \\
\hline & 4.0 & 0.22511 & 0.22773 & 0.22729 & 0.00091 & 0.00097 \\
\hline & 4.5 & 0.18781 & 0.19120 & 0.19212 & 0.00087 & 0.00097 \\
\hline & 5.0 & 0.15683 & 0.16081 & 0.16317 & 0.00082 & 0.00096 \\
\hline & 5.5 & 0.13109 & 0.13547 & 0.13920 & 0.00075 & 0.00096 \\
\hline \multirow{11}{*}{250} & 0.5 & 0.82617 & 0.82155 & 0.82664 & 0.00012 & 0.00051 \\
\hline & 1.0 & 0.68353 & 0.67869 & 0.68294 & 0.00028 & 0.00071 \\
\hline & 1.5 & 0.56628 & 0.56234 & 0.56410 & 0.00044 & 0.00077 \\
\hline & 2.0 & 0.46975 & 0.46709 & 0.46677 & 0.00058 & 0.00080 \\
\hline & 2.5 & 0.39017 & 0.38882 & 0.38741 & 0.00068 & 0.00082 \\
\hline & 3.0 & 0.32445 & 0.32433 & 0.32279 & 0.00074 & 0.00083 \\
\hline & 3.5 & 0.27011 & 0.27104 & 0.27010 & 0.00077 & 0.00082 \\
\hline & 4.0 & 0.22511 & 0.22692 & 0.22699 & 0.00076 & 0.00081 \\
\hline & 4.5 & 0.18781 & 0.19030 & 0.19160 & 0.00073 & 0.00080 \\
\hline & 5.0 & 0.15683 & 0.15986 & 0.16241 & 0.00068 & 0.00079 \\
\hline & 5.5 & 0.13109 & 0.13449 & 0.13823 & 0.00062 & 0.00078 \\
\hline
\end{tabular}


Table (7): values of reliability function estimator \& MSE for estimating reliability function by all methods for first model when $(\alpha=0.4, T=2)$ for $L=1000$.

\begin{tabular}{|c|c|c|c|c|c|c|}
\hline$n$ & $t_{\mathrm{i}}$ & Real & $M L E$ & WLS & $\begin{array}{l}M S E(\hat{R}) \\
M L E\end{array}$ & $\begin{array}{l}\text { MSE (R) } \\
W L S\end{array}$ \\
\hline \multirow{11}{*}{150} & 0.5 & 0.81940 & 0.80937 & 0.82114 & 0.00042 & 0.00086 \\
\hline & 1.0 & 0.67253 & 0.66507 & 0.66915 & 0.00076 & 0.00108 \\
\hline & 1.5 & 0.55286 & 0.55032 & 0.54973 & 0.00111 & 0.00126 \\
\hline & 2.0 & 0.45520 & 0.45786 & 0.45889 & 0.00147 & 0.00156 \\
\hline & 2.5 & 0.37536 & 0.38272 & 0.38885 & 0.00180 & 0.00218 \\
\hline & 3.0 & 0.30997 & 0.32125 & 0.33333 & 0.00206 & 0.00309 \\
\hline & 3.5 & 0.25635 & 0.27069 & 0.28825 & 0.00223 & 0.00410 \\
\hline & 4.0 & 0.21229 & 0.22890 & 0.25101 & 0.00232 & 0.00502 \\
\hline & 4.5 & 0.17603 & 0.19421 & 0.21985 & 0.00233 & 0.00577 \\
\hline & 5.0 & 0.14615 & 0.16531 & 0.19355 & 0.00229 & 0.00630 \\
\hline & 5.5 & 0.12149 & 0.14113 & 0.17118 & 0.00219 & 0.00661 \\
\hline \multirow{11}{*}{200} & 0.5 & 0.81940 & 0.81131 & 0.82099 & 0.00032 & 0.00068 \\
\hline & 1.0 & 0.67253 & 0.66696 & 0.67003 & 0.00061 & 0.00086 \\
\hline & 1.5 & 0.55286 & 0.55173 & 0.55104 & 0.00092 & 0.00104 \\
\hline & 2.0 & 0.45520 & 0.45866 & 0.45980 & 0.00124 & 0.00134 \\
\hline & 2.5 & 0.37536 & 0.38294 & 0.38916 & 0.00152 & 0.00192 \\
\hline & 3.0 & 0.30997 & 0.32095 & 0.33320 & 0.00173 & 0.00276 \\
\hline & 3.5 & 0.25635 & 0.26997 & 0.28788 & 0.00187 & 0.00369 \\
\hline & 4.0 & 0.21229 & 0.22784 & 0.25054 & 0.00194 & 0.00456 \\
\hline & 4.5 & 0.17603 & 0.19289 & 0.21937 & 0.00194 & 0.00526 \\
\hline & 5.0 & 0.14615 & 0.16379 & 0.19307 & 0.00189 & 0.00576 \\
\hline & 5.5 & 0.12149 & 0.13948 & 0.17072 & 0.00180 & 0.00606 \\
\hline \multirow{11}{*}{250} & 0.5 & 0.81940 & 0.81262 & 0.82080 & 0.00026 & 0.00056 \\
\hline & 1.0 & 0.67253 & 0.66757 & 0.67008 & 0.00052 & 0.00075 \\
\hline & 1.5 & 0.55286 & 0.55130 & 0.55075 & 0.00078 & 0.00091 \\
\hline & 2.0 & 0.45520 & 0.45720 & 0.45849 & 0.00103 & 0.00110 \\
\hline & 2.5 & 0.37536 & 0.38055 & 0.38669 & 0.00123 & 0.00147 \\
\hline & 3.0 & 0.30997 & 0.31780 & 0.32974 & 0.00138 & 0.00206 \\
\hline & 3.5 & 0.25635 & 0.26622 & 0.28367 & 0.00146 & 0.00276 \\
\hline & 4.0 & 0.21229 & 0.22365 & 0.24578 & 0.00148 & 0.00343 \\
\hline & 4.5 & 0.17603 & 0.18840 & 0.21421 & 0.00145 & 0.00399 \\
\hline & 5.0 & 0.14615 & 0.15911 & 0.18765 & 0.00139 & 0.00440 \\
\hline & 5.5 & 0.12149 & 0.13471 & 0.16511 & 0.00130 & 0.00464 \\
\hline
\end{tabular}


Table (8): values of reliability function estimator \& MSE for estimating reliability function by all methods for first model when $(\alpha=0.4, T=4)$ for $L=1000$.

\begin{tabular}{|c|c|c|c|c|c|c|}
\hline$n$ & $t_{\mathrm{i}}$ & Real & $M L E$ & WLS & $\begin{array}{l}\text { MSE }(\hat{R}) \\
M L E\end{array}$ & $\begin{array}{l}\text { MSE }(\hat{R}) \\
W L S\end{array}$ \\
\hline \multirow{11}{*}{150} & 0.5 & 0.81940 & 0.81206 & 0.81977 & 0.00022 & 0.00077 \\
\hline & 1.0 & 0.67253 & 0.66487 & 0.67146 & 0.00047 & 0.00104 \\
\hline & 1.5 & 0.55286 & 0.54672 & 0.54953 & 0.00070 & 0.00111 \\
\hline & 2.0 & 0.45520 & 0.45120 & 0.45063 & 0.00089 & 0.00115 \\
\hline & 2.5 & 0.37536 & 0.37358 & 0.37101 & 0.00103 & 0.00118 \\
\hline & 3.0 & 0.30997 & 0.31026 & 0.30705 & 0.00111 & 0.00119 \\
\hline & 3.5 & 0.25635 & 0.25841 & 0.25558 & 0.00115 & 0.00119 \\
\hline & 4.0 & 0.21229 & 0.21581 & 0.21400 & 0.00114 & 0.00119 \\
\hline & 4.5 & 0.17603 & 0.18070 & 0.18022 & 0.00110 & 0.00119 \\
\hline & 5.0 & 0.14615 & 0.15168 & 0.15260 & 0.00103 & 0.00118 \\
\hline & 5.5 & 0.12149 & 0.12764 & 0.12989 & 0.00095 & 0.00116 \\
\hline \multirow{11}{*}{200} & 0.5 & 0.81940 & 0.81414 & 0.81999 & 0.00017 & 0.00061 \\
\hline & 1.0 & 0.67253 & 0.66712 & 0.67211 & 0.00036 & 0.00084 \\
\hline & 1.5 & 0.55286 & 0.54861 & 0.55071 & 0.00055 & 0.00090 \\
\hline & 2.0 & 0.45520 & 0.45251 & 0.45211 & 0.00069 & 0.00093 \\
\hline & 2.5 & 0.37536 & 0.37428 & 0.37248 & 0.00079 & 0.00094 \\
\hline & 3.0 & 0.30997 & 0.31037 & 0.30827 & 0.00085 & 0.00095 \\
\hline & 3.5 & 0.25635 & 0.25800 & 0.25644 & 0.00087 & 0.00094 \\
\hline & 4.0 & 0.21229 & 0.21497 & 0.21444 & 0.00085 & 0.00093 \\
\hline & 4.5 & 0.17603 & 0.17952 & 0.18026 & 0.00081 & 0.00092 \\
\hline & 5.0 & 0.14615 & 0.15024 & 0.15229 & 0.00075 & 0.00091 \\
\hline & 5.5 & 0.12149 & 0.12600 & 0.12927 & 0.00068 & 0.00089 \\
\hline \multirow{11}{*}{250} & 0.5 & 0.81940 & 0.81447 & 0.81991 & 0.00014 & 0.00052 \\
\hline & 1.0 & 0.67253 & 0.66734 & 0.67202 & 0.00031 & 0.00071 \\
\hline & 1.5 & 0.55286 & 0.54865 & 0.55080 & 0.00047 & 0.00077 \\
\hline & 2.0 & 0.45520 & 0.45239 & 0.45233 & 0.00059 & 0.00081 \\
\hline & 2.5 & 0.37536 & 0.37400 & 0.37268 & 0.00068 & 0.00082 \\
\hline & 3.0 & 0.30997 & 0.30997 & 0.30830 & 0.00072 & 0.00082 \\
\hline & 3.5 & 0.25635 & 0.25750 & 0.25617 & 0.00073 & 0.00080 \\
\hline & 4.0 & 0.21229 & 0.21439 & 0.21383 & 0.00072 & 0.00079 \\
\hline & 4.5 & 0.17603 & 0.17888 & 0.17929 & 0.00068 & 0.00077 \\
\hline & 5.0 & 0.14615 & 0.14956 & 0.15099 & 0.00063 & 0.00075 \\
\hline & 5.5 & 0.12149 & 0.12529 & 0.12768 & 0.00058 & 0.00072 \\
\hline
\end{tabular}


Table (9): values of reliability function estimator \& MSE for estimating reliability function by all methods for first model when $(\alpha=0.5, T=2)$ for $L=1000$.

\begin{tabular}{|c|c|c|c|c|c|c|}
\hline$n$ & $t_{\mathrm{i}}$ & Real & $M L E$ & WLS & $\begin{array}{l}\text { MSE }(\hat{R}) \\
M L E\end{array}$ & $\begin{array}{l}\text { MSE }(\hat{R}) \\
W L S\end{array}$ \\
\hline \multirow{11}{*}{150} & 0.5 & 0.81264 & 0.80241 & 0.81419 & 0.00045 & 0.00088 \\
\hline & 1.0 & 0.66153 & 0.65387 & 0.65793 & 0.00079 & 0.00110 \\
\hline & 1.5 & 0.53944 & 0.53679 & 0.53626 & 0.00113 & 0.00128 \\
\hline & 2.0 & 0.44064 & 0.44329 & 0.44443 & 0.00146 & 0.00155 \\
\hline & 2.5 & 0.36055 & 0.36797 & 0.37417 & 0.00175 & 0.00215 \\
\hline & 3.0 & 0.29550 & 0.30688 & 0.31889 & 0.00198 & 0.00304 \\
\hline & 3.5 & 0.24258 & 0.25706 & 0.27432 & 0.00213 & 0.00400 \\
\hline & 4.0 & 0.19946 & 0.21620 & 0.23773 & 0.00220 & 0.00485 \\
\hline & 4.5 & 0.16426 & 0.18254 & 0.20731 & 0.00221 & 0.00551 \\
\hline & 5.0 & 0.13548 & 0.15469 & 0.18176 & 0.00215 & 0.00594 \\
\hline & 5.5 & 0.11190 & 0.13155 & 0.16014 & 0.00206 & 0.00616 \\
\hline \multirow{11}{*}{200} & 0.5 & 0.81264 & 0.80446 & 0.81398 & 0.00034 & 0.00070 \\
\hline & 1.0 & 0.66153 & 0.65580 & 0.65878 & 0.00063 & 0.00087 \\
\hline & 1.5 & 0.53944 & 0.53814 & 0.53740 & 0.00092 & 0.00105 \\
\hline & 2.0 & 0.44064 & 0.44396 & 0.44506 & 0.00121 & 0.00132 \\
\hline & 2.5 & 0.36055 & 0.36801 & 0.37419 & 0.00146 & 0.00187 \\
\hline & 3.0 & 0.29550 & 0.30638 & 0.31854 & 0.00165 & 0.00267 \\
\hline & 3.5 & 0.24258 & 0.25612 & 0.27381 & 0.00178 & 0.00355 \\
\hline & 4.0 & 0.19946 & 0.21493 & 0.23718 & 0.00183 & 0.00435 \\
\hline & 4.5 & 0.16426 & 0.18103 & 0.20678 & 0.00183 & 0.00498 \\
\hline & 5.0 & 0.13548 & 0.15302 & 0.18126 & 0.00178 & 0.00540 \\
\hline & 5.5 & 0.11190 & 0.12978 & 0.15966 & 0.00170 & 0.00563 \\
\hline \multirow{11}{*}{250} & 0.5 & 0.81264 & 0.80570 & 0.81379 & 0.00028 & 0.00059 \\
\hline & 1.0 & 0.66153 & 0.65656 & 0.65905 & 0.00053 & 0.00076 \\
\hline & 1.5 & 0.53944 & 0.53809 & 0.53770 & 0.00078 & 0.00090 \\
\hline & 2.0 & 0.44064 & 0.44307 & 0.44467 & 0.00102 & 0.00109 \\
\hline & 2.5 & 0.36055 & 0.36635 & 0.37280 & 0.00121 & 0.00147 \\
\hline & 3.0 & 0.29550 & 0.30408 & 0.31617 & 0.00135 & 0.00205 \\
\hline & 3.5 & 0.24258 & 0.25331 & 0.27064 & 0.00143 & 0.00272 \\
\hline & 4.0 & 0.19946 & 0.21174 & 0.23340 & 0.00146 & 0.00334 \\
\hline & 4.5 & 0.16426 & 0.17757 & 0.20252 & 0.00144 & 0.00384 \\
\hline & 5.0 & 0.13548 & 0.14938 & 0.17666 & 0.00138 & 0.00416 \\
\hline & 5.5 & 0.11190 & 0.12605 & 0.15480 & 0.00130 & 0.00433 \\
\hline
\end{tabular}


Table (10): values of reliability function estimator \& MSE for estimating reliability function by all methods for first model when $(\alpha=0.5, T=4)$ for $L=1000$.

\begin{tabular}{|c|c|c|c|c|c|c|}
\hline$n$ & $t_{\mathrm{i}}$ & Real & $M L E$ & WLS & $\begin{array}{l}M S E(\hat{R}) \\
M L E\end{array}$ & $\begin{array}{l}\text { MSE }(\hat{R}) \\
W L S\end{array}$ \\
\hline \multirow{11}{*}{150} & 0.5 & 0.81264 & 0.80455 & 0.81291 & 0.00028 & 0.00080 \\
\hline & 1.0 & 0.66153 & 0.65306 & 0.66013 & 0.00056 & 0.00107 \\
\hline & 1.5 & 0.53944 & 0.53271 & 0.53575 & 0.00078 & 0.00112 \\
\hline & 2.0 & 0.44064 & 0.43633 & 0.43587 & 0.00093 & 0.00115 \\
\hline & 2.5 & 0.36055 & 0.35874 & 0.35622 & 0.00103 & 0.00115 \\
\hline & 3.0 & 0.29550 & 0.29598 & 0.29282 & 0.00108 & 0.00115 \\
\hline & 3.5 & 0.24258 & 0.24501 & 0.24227 & 0.00109 & 0.00114 \\
\hline & 4.0 & 0.19946 & 0.20347 & 0.20177 & 0.00107 & 0.00114 \\
\hline & 4.5 & 0.16426 & 0.16948 & 0.16912 & 0.00102 & 0.00113 \\
\hline & 5.0 & 0.13548 & 0.14159 & 0.14261 & 0.00095 & 0.00112 \\
\hline & 5.5 & 0.11190 & 0.11862 & 0.12094 & 0.00088 & 0.00110 \\
\hline \multirow{11}{*}{200} & 0.5 & 0.81264 & 0.80681 & 0.81301 & 0.00020 & 0.00063 \\
\hline & 1.0 & 0.66153 & 0.65552 & 0.66074 & 0.00040 & 0.00085 \\
\hline & 1.5 & 0.53944 & 0.53477 & 0.53697 & 0.00058 & 0.00091 \\
\hline & 2.0 & 0.44064 & 0.43781 & 0.43742 & 0.00070 & 0.00093 \\
\hline & 2.5 & 0.36055 & 0.35959 & 0.35781 & 0.00078 & 0.00093 \\
\hline & 3.0 & 0.29550 & 0.29626 & 0.29422 & 0.00082 & 0.00092 \\
\hline & 3.5 & 0.24258 & 0.24481 & 0.24333 & 0.00082 & 0.00090 \\
\hline & 4.0 & 0.19946 & 0.20287 & 0.20243 & 0.00079 & 0.00088 \\
\hline & 4.5 & 0.16426 & 0.16857 & 0.16939 & 0.00075 & 0.00087 \\
\hline & 5.0 & 0.13548 & 0.14045 & 0.14253 & 0.00069 & 0.00085 \\
\hline & 5.5 & 0.11190 & 0.11732 & 0.12056 & 0.00063 & 0.00084 \\
\hline \multirow{11}{*}{250} & 0.5 & 0.81264 & 0.80744 & 0.81298 & 0.00016 & 0.00054 \\
\hline & 1.0 & 0.66153 & 0.65604 & 0.66096 & 0.00034 & 0.00073 \\
\hline & 1.5 & 0.53944 & 0.53500 & 0.53748 & 0.00049 & 0.00078 \\
\hline & 2.0 & 0.44064 & 0.43769 & 0.43800 & 0.00061 & 0.00080 \\
\hline & 2.5 & 0.36055 & 0.35915 & 0.35816 & 0.00067 & 0.00080 \\
\hline & 3.0 & 0.29550 & 0.29553 & 0.29414 & 0.00070 & 0.00079 \\
\hline & 3.5 & 0.24258 & 0.24384 & 0.24272 & 0.00070 & 0.00078 \\
\hline & 4.0 & 0.19946 & 0.20171 & 0.20129 & 0.00068 & 0.00075 \\
\hline & 4.5 & 0.16426 & 0.16728 & 0.16775 & 0.00064 & 0.00073 \\
\hline & 5.0 & 0.13548 & 0.13907 & 0.14047 & 0.00059 & 0.00071 \\
\hline & 5.5 & 0.11190 & 0.11589 & 0.11817 & 0.00053 & 0.00068 \\
\hline
\end{tabular}


Table (11): values of IMSE for estimating reliability function by all methods \& sample size \& for values of the mixed ratio parameter $(\alpha)$ for first model.

\begin{tabular}{|c|c|c|c|c|c|c|}
\hline$\alpha$ & $n$ & $T$ & $M$ & $M L E$ & WLS & Better \\
\hline \multirow{6}{*}{0.3} & \multirow[t]{2}{*}{150} & 2 & 4 & 0.00209 & 0.00378 & MLE \\
\hline & & 4 & 8 & 0.00092 & 0.00117 & MLE \\
\hline & \multirow[t]{2}{*}{200} & 2 & 4 & 0.00146 & 0.00324 & MLE \\
\hline & & 4 & 8 & 0.00069 & 0.00090 & MLE \\
\hline & \multirow[t]{2}{*}{250} & 2 & 4 & 0.00118 & 0.00274 & MLE \\
\hline & & 4 & 8 & 0.00058 & 0.00076 & MLE \\
\hline \multirow{6}{*}{0.4} & \multirow[t]{2}{*}{150} & 2 & 4 & 0.00172 & 0.00343 & MLE \\
\hline & & 4 & 8 & 0.00089 & 0.00112 & MLE \\
\hline & \multirow[t]{2}{*}{200} & 2 & 4 & 0.00143 & 0.00308 & MLE \\
\hline & & 4 & 8 & 0.00067 & 0.00088 & MLE \\
\hline & \multirow[t]{2}{*}{250} & 2 & 4 & 0.00111 & 0.00088 & MLE \\
\hline & & 4 & 8 & 0.00056 & 0.00075 & MLE \\
\hline \multirow{6}{*}{0.5} & \multirow[t]{2}{*}{150} & 2 & 4 & 0.00166 & 0.00331 & MLE \\
\hline & & 4 & 8 & 0.00087 & 0.00109 & MLE \\
\hline & \multirow[t]{2}{*}{200} & 2 & 4 & 0.00137 & 0.00294 & MLE \\
\hline & & 4 & 8 & 0.00065 & 0.00086 & MLE \\
\hline & \multirow[t]{2}{*}{250} & 2 & 4 & 0.00110 & 0.00229 & MLE \\
\hline & & 4 & 8 & 0.00055 & 0.00073 & MLE \\
\hline
\end{tabular}

Table (12): values of estimating mixed weibull distribution for two partial populations by MLE second model and $L=1000$.

\begin{tabular}{|c|c|c|c|c|c|c|c|c|}
\hline$\alpha$ & $n$ & $\bar{T}$ & $M$ & $p_{1}$ & $p_{1}$ & $\theta_{1}$ & $\theta_{2}$ & $\alpha$ \\
\hline \multirow{6}{*}{0.3} & \multirow[t]{2}{*}{150} & 2 & 4 & 0.85083 & 0.95918 & 2.70314 & 3.64857 & 0.31014 \\
\hline & & 4 & 8 & 0.85181 & 0.96055 & 2.75220 & 3.67153 & 0.31067 \\
\hline & \multirow[t]{2}{*}{200} & 2 & 4 & 0.85517 & 0.96091 & 2.61642 & 3.72012 & 0.30216 \\
\hline & & 4 & 8 & 0.85540 & 0.96118 & 2.76982 & 3.67155 & 0.31091 \\
\hline & \multirow[t]{2}{*}{250} & 2 & 4 & 0.85182 & 0.96263 & 2.73993 & 3.64435 & 0.31088 \\
\hline & & 4 & 8 & 0.85461 & 0.96399 & 2.72113 & 3.69736 & 0.30796 \\
\hline \multirow{6}{*}{0.4} & \multirow[t]{2}{*}{150} & 2 & 4 & 0.85537 & 0.96060 & 2.60652 & 3.71222 & 0.40432 \\
\hline & & 4 & 8 & 0.85590 & 0.96237 & 2.64290 & 3.72678 & 0.40565 \\
\hline & \multirow[t]{2}{*}{200} & 2 & 4 & 0.85763 & 0.96193 & 2.64254 & 3.68936 & 0.40524 \\
\hline & & 4 & 8 & 0.85903 & 0.96347 & 2.66647 & 3.72083 & 0.40554 \\
\hline & \multirow[t]{2}{*}{250} & 2 & 4 & 0.85569 & 0.96458 & 2.58630 & 3.73566 & 0.40157 \\
\hline & & 4 & 8 & 0.85741 & 0.96629 & 2.60757 & 3.38644 & 0.42245 \\
\hline \multirow{6}{*}{0.5} & \multirow[t]{2}{*}{150} & 2 & 4 & 0.85877 & 0.96150 & 2.54166 & 3.79105 & 0.49843 \\
\hline & & 4 & 8 & 0.85972 & 0.96303 & 2.57669 & 3.80505 & 0.50023 \\
\hline & \multirow[t]{2}{*}{200} & 2 & 4 & 0.86026 & 0.96200 & 2.54704 & 3.79551 & 0.49749 \\
\hline & & 4 & 8 & 0.86128 & 0.96468 & 2.58900 & 3.80436 & 0.50018 \\
\hline & \multirow[t]{2}{*}{250} & 2 & 4 & 0.85680 & 0.96681 & 2.51258 & 3.83237 & 0.49398 \\
\hline & & 4 & 8 & 0.85912 & 0.96932 & 2.54304 & 3.85739 & 0.49560 \\
\hline
\end{tabular}


Table (13): values of estimating mixed weibull distribution for two partial populations by WLS second model and $\mathrm{L}=1000$.

\begin{tabular}{|c|c|c|c|c|c|c|c|c|}
\hline$\alpha$ & $n$ & $T$ & $M$ & $p_{1}$ & $p_{2}$ & $\theta_{1}$ & $\theta_{2}$ & $\alpha$ \\
\hline \multirow{6}{*}{0.3} & \multirow{2}{*}{150} & 2 & 4 & 0.83083 & 0.96980 & 3.01024 & 3.24068 & 0.35758 \\
\hline & & 4 & 8 & 0.82058 & 0.95691 & 2.88809 & 3.32441 & 0.34417 \\
\hline & \multirow[t]{2}{*}{200} & 2 & 4 & 0.83307 & 0.95138 & 2.94055 & 3.25929 & 0.34749 \\
\hline & & 4 & 8 & 0.82208 & 0.94237 & 2.84502 & 3.32005 & 0.33799 \\
\hline & \multirow[t]{2}{*}{250} & 2 & 4 & 0.82669 & 0.94472 & 2.91897 & 3.25208 & 0.34522 \\
\hline & & 4 & 8 & 0.82021 & 0.93544 & 2.79101 & 3.33010 & 0.33269 \\
\hline \multirow{6}{*}{0.4} & \multirow[t]{2}{*}{150} & 2 & 4 & 0.83410 & 0.97167 & 2.71349 & 3.33052 & 0.43947 \\
\hline & & 4 & 8 & 0.82376 & 0.95808 & 2.65555 & 3.39218 & 0.43113 \\
\hline & \multirow[t]{2}{*}{200} & 2 & 4 & 0.83221 & 0.95267 & 2.70368 & 3.31326 & 0.43292 \\
\hline & & 4 & 8 & 0.82382 & 0.94233 & 2.65532 & 3.36314 & 0.42633 \\
\hline & \multirow[t]{2}{*}{250} & 2 & 4 & 0.82422 & 0.94664 & 2.67425 & 3.32100 & 0.43114 \\
\hline & & 4 & 8 & 0.81828 & 0.93664 & 2.60706 & 3.38644 & 0.42245 \\
\hline \multirow{6}{*}{0.5} & \multirow[t]{2}{*}{150} & 2 & 4 & 0.83370 & 0.97528 & 2.55931 & 3.43685 & 0.52422 \\
\hline & & 4 & 8 & 0.82504 & 0.96184 & 2.54001 & 3.47235 & 0.52035 \\
\hline & \multirow[t]{2}{*}{200} & 2 & 4 & 0.83506 & 0.95106 & 2.52500 & 3.43756 & 0.51566 \\
\hline & & 4 & 8 & 0.82682 & 0.94141 & 2.51762 & 3.45271 & 0.51420 \\
\hline & \multirow[t]{2}{*}{250} & 2 & 4 & 0.82324 & 0.94755 & 2.52734 & 3.41070 & 0.51679 \\
\hline & & 4 & 8 & 0.81747 & 0.93776 & 2.50000 & 3.45022 & 0.51227 \\
\hline
\end{tabular}

Table (14): values of MSE for mixed weibull distribution for two partial populations ny MLE method second model $(\mathrm{L}=1000)$.

\begin{tabular}{|c|c|c|c|c|c|c|c|c|}
\hline$\alpha$ & $n$ & $T$ & $M$ & $p_{1}$ & $p_{1}$ & $\theta_{1}$ & $\theta_{2}$ & $\alpha$ \\
\hline \multirow{6}{*}{0.3} & \multirow[t]{2}{*}{150} & 2 & 4 & 0.01716 & 0.00911 & 3.44844 & 1.59889 & 0.02012 \\
\hline & & 4 & 8 & 0.00848 & 0.00665 & 1.99538 & 0.93639 & 0.00667 \\
\hline & \multirow[t]{2}{*}{200} & 2 & 4 & 0.00947 & 0.00637 & 22.40606 & 7.47049 & 0.10133 \\
\hline & & 4 & 8 & 0.00759 & 0.00608 & 1.09761 & 0.48832 & 0.00281 \\
\hline & \multirow[t]{2}{*}{250} & 2 & 4 & 0.00813 & 0.00630 & 1.25303 & 0.54869 & 0.00392 \\
\hline & & 4 & 8 & 0.00678 & 0.00604 & 0.93396 & 0.41167 & 0.00227 \\
\hline \multirow{6}{*}{0.4} & \multirow[t]{2}{*}{150} & 2 & 4 & 0.00925 & 0.00727 & 1.05806 & 0.88280 & 0.00509 \\
\hline & & 4 & 8 & 0.00782 & 0.00697 & 0.83419 & 0.71882 & 0.00325 \\
\hline & \multirow[t]{2}{*}{200} & 2 & 4 & 0.00850 & 0.00668 & 0.94775 & 0.76398 & 0.00455 \\
\hline & & 4 & 8 & 0.00716 & 0.00639 & 0.73416 & 0.57650 & 0.00268 \\
\hline & \multirow[t]{2}{*}{250} & 2 & 4 & 0.00770 & 0.00673 & 0.84608 & 0.60749 & 0.00373 \\
\hline & & 4 & 8 & 0.00672 & 0.00643 & 0.65168 & 0.47362 & 0.00223 \\
\hline \multirow{6}{*}{0.5} & \multirow[t]{2}{*}{150} & 2 & 4 & 0.00890 & 0.00777 & 0.83649 & 1.18197 & 0.00527 \\
\hline & & 4 & 8 & 0.00769 & 0.00736 & 0.68527 & 0.94582 & 0.00337 \\
\hline & \multirow[t]{2}{*}{200} & 2 & 4 & 0.00801 & 0.00692 & 0.75125 & 0.97779 & 0.00453 \\
\hline & & 4 & 8 & 0.00695 & 0.00672 & 0.59347 & 0.75993 & 0.00274 \\
\hline & \multirow[t]{2}{*}{250} & 2 & 4 & 0.00717 & 0.00716 & 0.69686 & 0.80098 & 0.00396 \\
\hline & & 4 & 8 & 0.00636 & 0.00689 & 0.54720 & 0.60570 & 0.00229 \\
\hline
\end{tabular}


Table (15): MSE values of mixed weibull distribution for two partial populations by WLS second model ( $L=1000)$.

\begin{tabular}{|c|c|c|c|c|c|c|c|c|}
\hline$\alpha$ & $n$ & $T$ & $M$ & $p_{1}$ & $p_{1}$ & $\theta_{1}$ & $\theta_{2}$ & $\alpha$ \\
\hline \multirow{6}{*}{0.3} & \multirow[t]{2}{*}{150} & 2 & 4 & 0.13303 & 0.09953 & 5.23190 & 3.07919 & 0.05253 \\
\hline & & 4 & 8 & 0.04193 & 0.02651 & 1.20842 & 1.08556 & 0.00939 \\
\hline & \multirow[t]{2}{*}{200} & 2 & 4 & 0.04465 & 0.02516 & 1.54986 & 1.15345 & 0.01295 \\
\hline & & 4 & 8 & 0.03532 & 0.01912 & 1.07146 & 0.92618 & 0.00807 \\
\hline & \multirow[t]{2}{*}{250} & 2 & 4 & 0.03663 & 0.02047 & 1.35565 & 1.08392 & 0.01177 \\
\hline & & 4 & 8 & 0.02939 & 0.01577 & 0.92537 & 0.83113 & 0.00686 \\
\hline \multirow{6}{*}{0.4} & \multirow[t]{2}{*}{150} & 2 & 4 & 0.03995 & 0.04012 & 1.02775 & 1.46490 & 0.01325 \\
\hline & & 4 & 8 & 0.03192 & 0.03103 & 0.78395 & 1.14296 & 0.00806 \\
\hline & \multirow[t]{2}{*}{200} & 2 & 4 & 0.03247 & 0.03074 & 0.97615 & 1.23154 & 0.01141 \\
\hline & & 4 & 8 & 0.02597 & 0.02341 & 0.73657 & 0.97491 & 0.00704 \\
\hline & \multirow[t]{2}{*}{250} & 2 & 4 & 0.02670 & 0.02295 & 0.86521 & 1.13034 & 0.01024 \\
\hline & & 4 & 8 & 0.02175 & 0.01768 & 0.64576 & 0.86592 & 0.00588 \\
\hline \multirow{6}{*}{0.5} & \multirow[t]{2}{*}{150} & 2 & 4 & 0.03145 & 0.04634 & 0.82091 & 1.71193 & 0.01145 \\
\hline & & 4 & 8 & 0.02471 & 0.03634 & 0.67698 & 1.34371 & 0.00718 \\
\hline & \multirow[t]{2}{*}{200} & 2 & 4 & 0.02645 & 0.03560 & 0.79419 & 1.37236 & 0.01007 \\
\hline & & 4 & 8 & 0.02130 & 0.02740 & 0.63514 & 1.07217 & 0.00625 \\
\hline & \multirow[t]{2}{*}{250} & 2 & 4 & 0.02041 & 0.02748 & 0.71431 & 1.26099 & 0.00906 \\
\hline & & 4 & 8 & 0.01638 & 0.02163 & 0.58227 & 0.92303 & 0.00528 \\
\hline
\end{tabular}


Table (16): estimating values of reliability distribution \& MSE for all methods second model when $(\alpha=0.3, T=4, L=1000)$.

\begin{tabular}{|c|c|c|c|c|c|c|}
\hline$n$ & $t_{\mathrm{i}}$ & Real & $M L E$ & WLS & $\begin{array}{l}M S E(\hat{R}) \\
M L E\end{array}$ & $\begin{array}{l}\text { MSE }(\hat{R}) \\
W L S\end{array}$ \\
\hline \multirow{11}{*}{150} & 0.5 & 0.85995 & 0.84469 & 0.83716 & 0.00055 & 0.00129 \\
\hline & 1.0 & 0.76011 & 0.73045 & 0.72141 & 0.00135 & 0.00249 \\
\hline & 1.5 & 0.67751 & 0.63656 & 0.62714 & 0.00233 & 0.00362 \\
\hline & 2.0 & 0.60693 & 0.55757 & 0.54923 & 0.00329 & 0.00449 \\
\hline & 2.5 & 0.54566 & 0.49028 & 0.48435 & 0.00412 & 0.00500 \\
\hline & 3.0 & 0.49192 & 0.43250 & 0.42998 & 0.00477 & 0.00516 \\
\hline & 3.5 & 0.44445 & 0.38260 & 0.38407 & 0.00522 & 0.00504 \\
\hline & 4.0 & 0.40231 & 0.33930 & 0.34502 & 0.00550 & 0.00476 \\
\hline & 4.5 & 0.36475 & 0.30156 & 0.31156 & 0.00562 & 0.00439 \\
\hline & 5.0 & 0.33115 & 0.26858 & 0.28268 & 0.00563 & 0.00401 \\
\hline & 5.5 & 0.30101 & 0.23966 & 0.25757 & 0.00554 & 0.00365 \\
\hline \multirow{11}{*}{200} & 0.5 & 0.85995 & 0.84709 & 0.83723 & 0.00034 & 0.00112 \\
\hline & 1.0 & 0.76011 & 0.73288 & 0.72197 & 0.00108 & 0.00224 \\
\hline & 1.5 & 0.67751 & 0.63859 & 0.62840 & 0.00203 & 0.00328 \\
\hline & 2.0 & 0.60693 & 0.55905 & 0.55103 & 0.00298 & 0.00407 \\
\hline & 2.5 & 0.54566 & 0.49117 & 0.48642 & 0.00383 & 0.00453 \\
\hline & 3.0 & 0.49192 & 0.43282 & 0.43204 & 0.00450 & 0.00468 \\
\hline & 3.5 & 0.44445 & 0.38238 & 0.38594 & 0.00497 & 0.00459 \\
\hline & 4.0 & 0.40231 & 0.33859 & 0.34655 & 0.00527 & 0.00434 \\
\hline & 4.5 & 0.36475 & 0.30042 & 0.31268 & 0.00541 & 0.00402 \\
\hline & 5.0 & 0.33115 & 0.26706 & 0.28335 & 0.00541 & 0.00367 \\
\hline & 5.5 & 0.30101 & 0.23782 & 0.25778 & 0.00533 & 0.00333 \\
\hline \multirow{11}{*}{250} & 0.5 & 0.85995 & 0.84753 & 0.83710 & 0.00030 & 0.00102 \\
\hline & 1.0 & 0.76011 & 0.73336 & 0.72198 & 0.00099 & 0.00213 \\
\hline & 1.5 & 0.67751 & 0.63898 & 0.62863 & 0.00191 & 0.00315 \\
\hline & 2.0 & 0.60693 & 0.55929 & 0.55136 & 0.00285 & 0.00391 \\
\hline & 2.5 & 0.54566 & 0.49124 & 0.48669 & 0.00369 & 0.00436 \\
\hline & 3.0 & 0.49192 & 0.43270 & 0.43211 & 0.00437 & 0.00451 \\
\hline & 3.5 & 0.44445 & 0.38208 & 0.38572 & 0.00486 & 0.00444 \\
\hline & 4.0 & 0.40231 & 0.33813 & 0.34599 & 0.00517 & 0.00421 \\
\hline & 4.5 & 0.36475 & 0.29984 & 0.31176 & 0.00532 & 0.00390 \\
\hline & 5.0 & 0.33115 & 0.26637 & 0.28208 & 0.00533 & 0.00356 \\
\hline & 5.5 & 0.30101 & 0.23706 & 0.25620 & 0.00523 & 0.00323 \\
\hline
\end{tabular}


Table (17): estimating values of reliability distribution \& MSE for all methods second model when $(\alpha=0.3, T=5, L=1000)$.

\begin{tabular}{|c|c|c|c|c|c|c|}
\hline$n$ & $t_{\mathrm{i}}$ & Real & $M L E$ & WLS & $\begin{array}{l}M S E(\hat{R}) \\
M L E\end{array}$ & $\begin{array}{l}\text { MSE }(\hat{R}) \\
W L S\end{array}$ \\
\hline \multirow{11}{*}{150} & 0.5 & 0.85995 & 0.84747 & 0.83677 & 0.00034 & 0.00128 \\
\hline & 1.0 & 0.76011 & 0.73361 & 0.72182 & 0.00107 & 0.00246 \\
\hline & 1.5 & 0.67751 & 0.63955 & 0.62792 & 0.00201 & 0.00352 \\
\hline & 2.0 & 0.60693 & 0.56015 & 0.54970 & 0.00298 & 0.00439 \\
\hline & 2.5 & 0.54566 & 0.49236 & 0.48393 & 0.00385 & 0.00498 \\
\hline & 3.0 & 0.49192 & 0.43405 & 0.42828 & 0.00454 & 0.00528 \\
\hline & 3.5 & 0.44445 & 0.38364 & 0.38093 & 0.00504 & 0.00531 \\
\hline & 4.0 & 0.40231 & 0.33985 & 0.34042 & 0.00535 & 0.00515 \\
\hline & 4.5 & 0.36475 & 0.30169 & 0.30558 & 0.00548 & 0.00486 \\
\hline & 5.0 & 0.33115 & 0.26833 & 0.27546 & 0.00548 & 0.00450 \\
\hline & 5.5 & 0.30101 & 0.23909 & 0.24928 & 0.00537 & 0.00411 \\
\hline \multirow{11}{*}{200} & 0.5 & 0.85995 & 0.84879 & 0.83698 & 0.00027 & 0.00111 \\
\hline & 1.0 & 0.76011 & 0.73522 & 0.72235 & 0.00089 & 0.00222 \\
\hline & 1.5 & 0.67751 & 0.64114 & 0.62900 & 0.00174 & 0.00322 \\
\hline & 2.0 & 0.60693 & 0.56156 & 0.55126 & 0.00264 & 0.00401 \\
\hline & 2.5 & 0.54566 & 0.49351 & 0.48580 & 0.00345 & 0.00454 \\
\hline & 3.0 & 0.49192 & 0.43489 & 0.43028 & 0.00412 & 0.00480 \\
\hline & 3.5 & 0.44445 & 0.38414 & 0.38288 & 0.00462 & 0.00483 \\
\hline & 4.0 & 0.40231 & 0.34002 & 0.34220 & 0.00494 & 0.00468 \\
\hline & 4.5 & 0.36475 & 0.30155 & 0.30710 & 0.00510 & 0.00442 \\
\hline & 5.0 & 0.33115 & 0.26790 & 0.27666 & 0.00512 & 0.00409 \\
\hline & 5.5 & 0.30101 & 0.23840 & 0.25013 & 0.00504 & 0.00374 \\
\hline \multirow{11}{*}{250} & 0.5 & 0.85995 & 0.84966 & 0.83690 & 0.00022 & 0.00102 \\
\hline & 1.0 & 0.76011 & 0.73608 & 0.72233 & 0.00080 & 0.00210 \\
\hline & 1.5 & 0.67751 & 0.64181 & 0.62914 & 0.00162 & 0.00308 \\
\hline & 2.0 & 0.60693 & 0.56195 & 0.55151 & 0.00251 & 0.00386 \\
\hline & 2.5 & 0.54566 & 0.49358 & 0.48606 & 0.00333 & 0.00438 \\
\hline & 3.0 & 0.49192 & 0.43464 & 0.43044 & 0.00402 & 0.00464 \\
\hline & 3.5 & 0.44445 & 0.38358 & 0.38287 & 0.00454 & 0.00468 \\
\hline & 4.0 & 0.40231 & 0.33918 & 0.34194 & 0.00489 & 0.00455 \\
\hline & 4.5 & 0.36475 & 0.30045 & 0.30656 & 0.00508 & 0.00431 \\
\hline & 5.0 & 0.33115 & 0.26657 & 0.27582 & 0.00514 & 0.00400 \\
\hline & 5.5 & 0.30101 & 0.23688 & 0.24900 & 0.00508 & 0.00366 \\
\hline
\end{tabular}


Table (18): estimating values of reliability distribution \& MSE for all methods second model when $(\alpha=0.4, T=4, L=1000)$.

\begin{tabular}{|c|c|c|c|c|c|c|}
\hline$n$ & $t_{\mathrm{i}}$ & Real & $M L E$ & WLS & $\begin{array}{l}\text { MSE }(\hat{R}) \\
M L E\end{array}$ & $\begin{array}{l}\text { MSE }(\hat{R}) \\
W L S\end{array}$ \\
\hline \multirow{11}{*}{150} & 0.5 & 0.85507 & 0.83872 & 0.83007 & 0.00051 & 0.00140 \\
\hline & 1.0 & 0.75389 & 0.72189 & 0.71219 & 0.00144 & 0.00273 \\
\hline & 1.5 & 0.67081 & 0.62673 & 0.61719 & 0.00256 & 0.00395 \\
\hline & 2.0 & 0.60018 & 0.54722 & 0.53923 & 0.00364 & 0.00487 \\
\hline & 2.5 & 0.53909 & 0.47988 & 0.47465 & 0.00455 & 0.00538 \\
\hline & 3.0 & 0.48566 & 0.42234 & 0.42073 & 0.00523 & 0.00552 \\
\hline & 3.5 & 0.43857 & 0.37286 & 0.37533 & 0.00569 & 0.00539 \\
\hline & 4.0 & 0.39685 & 0.33007 & 0.33679 & 0.00593 & 0.00507 \\
\hline & 4.5 & 0.35970 & 0.29292 & 0.30380 & 0.00600 & 0.00468 \\
\hline & 5.0 & 0.32652 & 0.26053 & 0.27534 & 0.00593 & 0.00426 \\
\hline & 5.5 & 0.29680 & 0.23222 & 0.25059 & 0.00575 & 0.00386 \\
\hline \multirow{11}{*}{200} & 0.5 & 0.85507 & 0.84052 & 0.83021 & 0.00040 & 0.00124 \\
\hline & 1.0 & 0.75389 & 0.72391 & 0.71287 & 0.00125 & 0.00250 \\
\hline & 1.5 & 0.67081 & 0.62860 & 0.61855 & 0.00230 & 0.00362 \\
\hline & 2.0 & 0.60018 & 0.54879 & 0.54106 & 0.00334 & 0.00445 \\
\hline & 2.5 & 0.53909 & 0.48108 & 0.47666 & 0.00423 & 0.00491 \\
\hline & 3.0 & 0.48566 & 0.42314 & 0.42266 & 0.00491 & 0.00505 \\
\hline & 3.5 & 0.43857 & 0.37326 & 0.37701 & 0.00538 & 0.00495 \\
\hline & 4.0 & 0.39685 & 0.33010 & 0.33811 & 0.00566 & 0.00468 \\
\hline & 4.5 & 0.35970 & 0.29261 & 0.30470 & 0.00575 & 0.00434 \\
\hline & 5.0 & 0.32652 & 0.25992 & 0.27581 & 0.00571 & 0.00396 \\
\hline & 5.5 & 0.29680 & 0.23134 & 0.25066 & 0.00556 & 0.00360 \\
\hline \multirow{11}{*}{250} & 0.5 & 0.85507 & 0.84123 & 0.83007 & 0.00035 & 0.00114 \\
\hline & 1.0 & 0.75389 & 0.72480 & 0.71298 & 0.00114 & 0.00236 \\
\hline & 1.5 & 0.67081 & 0.62944 & 0.61894 & 0.00215 & 0.00346 \\
\hline & 2.0 & 0.60018 & 0.54942 & 0.54154 & 0.00317 & 0.00427 \\
\hline & 2.5 & 0.53909 & 0.48142 & 0.47702 & 0.00406 & 0.00475 \\
\hline & 3.0 & 0.48566 & 0.42316 & 0.42272 & 0.00477 & 0.00491 \\
\hline & 3.5 & 0.43857 & 0.37295 & 0.37664 & 0.00527 & 0.00483 \\
\hline & 4.0 & 0.39685 & 0.32946 & 0.33726 & 0.00558 & 0.00459 \\
\hline & 4.5 & 0.35970 & 0.29166 & 0.30337 & 0.00571 & 0.00427 \\
\hline & 5.0 & 0.32652 & 0.25870 & 0.27400 & 0.00570 & 0.00390 \\
\hline & 5.5 & 0.29680 & 0.22987 & 0.24840 & 0.00558 & 0.00353 \\
\hline
\end{tabular}


Table (19): estimating values of reliability distribution \& MSE for all methods second model when $(\alpha=0,4, T=5, L=1000)$.

\begin{tabular}{|c|c|c|c|c|c|c|}
\hline$n$ & $t_{i}$ & Real & $M L E$ & WLS & $\begin{array}{l}M S E(\hat{R}) \\
M L E\end{array}$ & $\begin{array}{l}\text { MSE }(\hat{R}) \\
W L S\end{array}$ \\
\hline \multirow{11}{*}{150} & 0.5 & 0.85507 & 0.84116 & 0.82968 & 0.00038 & 0.00140 \\
\hline & 1.0 & 0.75389 & 0.72489 & 0.71264 & 0.00118 & 0.00269 \\
\hline & 1.5 & 0.67081 & 0.62974 & 0.61800 & 0.00221 & 0.00385 \\
\hline & 2.0 & 0.60018 & 0.54995 & 0.53968 & 0.00325 & 0.00477 \\
\hline & 2.5 & 0.53909 & 0.48220 & 0.47416 & 0.00416 & 0.00538 \\
\hline & 3.0 & 0.48566 & 0.42418 & 0.41894 & 0.00488 & 0.00566 \\
\hline & 3.5 & 0.43857 & 0.37420 & 0.37210 & 0.00539 & 0.00568 \\
\hline & 4.0 & 0.39685 & 0.33095 & 0.33212 & 0.00569 & 0.00549 \\
\hline & 4.5 & 0.35970 & 0.29335 & 0.29780 & 0.00582 & 0.00517 \\
\hline & 5.0 & 0.32652 & 0.26058 & 0.26817 & 0.00580 & 0.00477 \\
\hline & 5.5 & 0.29680 & 0.23191 & 0.24243 & 0.00566 & 0.00435 \\
\hline \multirow{11}{*}{200} & 0.5 & 0.85507 & 0.84292 & 0.82995 & 0.00030 & 0.00123 \\
\hline & 1.0 & 0.75389 & 0.72695 & 0.71324 & 0.00101 & 0.00246 \\
\hline & 1.5 & 0.67081 & 0.63173 & 0.61913 & 0.00196 & 0.00355 \\
\hline & 2.0 & 0.60018 & 0.55168 & 0.54126 & 0.00294 & 0.00439 \\
\hline & 2.5 & 0.53909 & 0.48357 & 0.47602 & 0.00382 & 0.00493 \\
\hline & 3.0 & 0.48566 & 0.42516 & 0.42089 & 0.00453 & 0.00519 \\
\hline & 3.5 & 0.43857 & 0.37478 & 0.37399 & 0.00504 & 0.00520 \\
\hline & 4.0 & 0.39685 & 0.33112 & 0.33383 & 0.00537 & 0.00502 \\
\hline & 4.5 & 0.35970 & 0.29315 & 0.29926 & 0.00552 & 0.00473 \\
\hline & 5.0 & 0.32652 & 0.26003 & 0.26933 & 0.00553 & 0.00438 \\
\hline & 5.5 & 0.29680 & 0.23105 & 0.24328 & 0.00542 & 0.00399 \\
\hline \multirow{11}{*}{250} & 0.5 & 0.85507 & 0.84340 & 0.82986 & 0.00027 & 0.00114 \\
\hline & 1.0 & 0.75389 & 0.72754 & 0.71332 & 0.00094 & 0.00232 \\
\hline & 1.5 & 0.67081 & 0.63226 & 0.61945 & 0.00186 & 0.00339 \\
\hline & 2.0 & 0.60018 & 0.55207 & 0.54172 & 0.00282 & 0.00421 \\
\hline & 2.5 & 0.53909 & 0.48376 & 0.47646 & 0.00370 & 0.00476 \\
\hline & 3.0 & 0.48566 & 0.42514 & 0.42116 & 0.00442 & 0.00502 \\
\hline & 3.5 & 0.43857 & 0.37453 & 0.37397 & 0.00494 & 0.00506 \\
\hline & 4.0 & 0.39685 & 0.33065 & 0.33346 & 0.00529 & 0.00493 \\
\hline & 4.5 & 0.35970 & 0.29248 & 0.29848 & 0.00546 & 0.00468 \\
\hline & 5.0 & 0.32652 & 0.25917 & 0.26812 & 0.00550 & 0.00435 \\
\hline & 5.5 & 0.29680 & 0.23002 & 0.24165 & 0.00541 & 0.00399 \\
\hline
\end{tabular}


Table (20): estimating values of reliability distribution \& MSE for all methods second model when $(\alpha=0.5, T=4, L=1000)$.

\begin{tabular}{|c|c|c|c|c|c|c|}
\hline$n$ & $t_{\mathrm{i}}$ & Real & $M L E$ & WLS & $\begin{array}{l}M S E(\hat{R}) \\
M L E\end{array}$ & $\begin{array}{l}\text { MSE }(\hat{R}) \\
W L S\end{array}$ \\
\hline \multirow{11}{*}{150} & 0.5 & 0.85018 & 0.83209 & 0.82298 & 0.00060 & 0.00154 \\
\hline & 1.0 & 0.74766 & 0.71277 & 0.70305 & 0.00168 & 0.00300 \\
\hline & 1.5 & 0.66411 & 0.61656 & 0.60738 & 0.00292 & 0.00431 \\
\hline & 2.0 & 0.59343 & 0.53677 & 0.52934 & 0.00408 & 0.00526 \\
\hline & 2.5 & 0.53252 & 0.46961 & 0.46496 & 0.00502 & 0.00579 \\
\hline & 3.0 & 0.47940 & 0.41252 & 0.41135 & 0.00570 & 0.00592 \\
\hline & 3.5 & 0.43269 & 0.36362 & 0.36629 & 0.00613 & 0.00577 \\
\hline & 4.0 & 0.39138 & 0.32149 & 0.32809 & 0.00634 & 0.00545 \\
\hline & 4.5 & 0.35466 & 0.28502 & 0.29542 & 0.00637 & 0.00503 \\
\hline & 5.0 & 0.32190 & 0.25331 & 0.26725 & 0.00625 & 0.00459 \\
\hline & 5.5 & 0.29258 & 0.22565 & 0.24278 & 0.00603 & 0.00416 \\
\hline \multirow{11}{*}{200} & 0.5 & 0.85018 & 0.83386 & 0.82295 & 0.00048 & 0.00139 \\
\hline & 1.0 & 0.74766 & 0.71486 & 0.70347 & 0.00145 & 0.00278 \\
\hline & 1.5 & 0.66411 & 0.61859 & 0.60843 & 0.00261 & 0.00400 \\
\hline & 2.0 & 0.59343 & 0.53857 & 0.53090 & 0.00372 & 0.00487 \\
\hline & 2.5 & 0.53252 & 0.47107 & 0.46681 & 0.00465 & 0.00534 \\
\hline & 3.0 & 0.47940 & 0.41359 & 0.41330 & 0.00535 & 0.00546 \\
\hline & 3.5 & 0.43269 & 0.36431 & 0.36819 & 0.00580 & 0.00531 \\
\hline & 4.0 & 0.39138 & 0.32180 & 0.32985 & 0.00604 & 0.00500 \\
\hline & 4.5 & 0.35466 & 0.28498 & 0.29700 & 0.00610 & 0.00461 \\
\hline & 5.0 & 0.32190 & 0.25296 & 0.26862 & 0.00602 & 0.00420 \\
\hline & 5.5 & 0.29258 & 0.22501 & 0.24394 & 0.00583 & 0.00379 \\
\hline \multirow{11}{*}{250} & 0.5 & 085018 & 083448 & 0.82276 & 000043 & 000129 \\
\hline & 1.0 & 0.74766 & 0.71569 & 0.70367 & 0.00134 & 0.00263 \\
\hline & 1.5 & 0.66411 & 0.61939 & 0.60904 & 0.00246 & 0.00381 \\
\hline & 2.0 & 0.59343 & 0.53918 & 0.53168 & 0.00355 & 0.00465 \\
\hline & 2.5 & 0.53252 & 0.47142 & 0.46751 & 0.00448 & 0.00512 \\
\hline & 3.0 & 0.47940 & 0.41365 & 0.41371 & 0.00519 & 0.00525 \\
\hline & 3.5 & 0.43269 & 0.36405 & 0.36820 & 0.00567 & 0.00514 \\
\hline & 4.0 & 0.39138 & 0.32124 & 0.32940 & 0.00594 & 0.00487 \\
\hline & 4.5 & 0.35466 & 0.28414 & 0.29606 & 0.00604 & 0.00450 \\
\hline & 5.0 & 0.32190 & 0.25186 & 0.26723 & 0.00599 & 0.00409 \\
\hline & 5.5 & 0.29258 & 0.22368 & 0.24213 & 0.00582 & 0.00369 \\
\hline
\end{tabular}


Table (21): estimating values of reliability distribution \& MSE for all methods second model when $(\alpha=0.5, T=5, L=1000)$.

\begin{tabular}{|c|c|c|c|c|c|c|}
\hline$n$ & $t_{\mathrm{i}}$ & Real & MLE & WLS & $\begin{array}{l}M S E(\hat{R}) \\
M L E\end{array}$ & $\begin{array}{l}\text { MSE }(\hat{R}) \\
W L S\end{array}$ \\
\hline \multirow{11}{*}{150} & 0.5 & 0.85018 & 0.83459 & 0.82263 & 0.00047 & 0.00153 \\
\hline & 1.0 & 0.74766 & 0.71588 & 0.70343 & 0.00140 & 0.00296 \\
\hline & 1.5 & 0.66411 & 0.61968 & 0.60806 & 0.00254 & 0.00422 \\
\hline & 2.0 & 0.59343 & 0.53962 & 0.52968 & 0.00366 & 0.00518 \\
\hline & 2.5 & 0.53252 & 0.47202 & 0.46442 & 0.00461 & 0.00579 \\
\hline & 3.0 & 0.47940 & 0.41442 & 0.40962 & 0.00533 & 0.00607 \\
\hline & 3.5 & 0.43269 & 0.36500 & 0.36326 & 0.00582 & 0.00606 \\
\hline & 4.0 & 0.39138 & 0.32238 & 0.32379 & 0.00609 & 0.00585 \\
\hline & 4.5 & 0.35466 & 0.28544 & 0.28996 & 0.00618 & 0.00550 \\
\hline & 5.0 & 0.32190 & 0.25332 & 0.26079 & 0.00612 & 0.00508 \\
\hline & 5.5 & 0.29258 & 0.22530 & 0.23548 & 0.00595 & 0.00463 \\
\hline \multirow{11}{*}{200} & 0.5 & 0.85018 & 0.83635 & 0.82274 & 0.00037 & 0.00138 \\
\hline & 1.0 & 0.74766 & 0.71797 & 0.70383 & 0.00120 & 0.00275 \\
\hline & 1.5 & 0.66411 & 0.62172 & 0.60895 & 0.00226 & 0.00394 \\
\hline & 2.0 & 0.59343 & 0.54141 & 0.53101 & 0.00333 & 0.00483 \\
\hline & 2.5 & 0.53252 & 0.47347 & 0.46608 & 0.00425 & 0.00538 \\
\hline & 3.0 & 0.47940 & 0.41549 & 0.41145 & 0.00497 & 0.00561 \\
\hline & 3.5 & 0.43269 & 0.36569 & 0.36514 & 0.00546 & 0.00558 \\
\hline & 4.0 & 0.39138 & 0.32268 & 0.32561 & 0.00576 & 0.00537 \\
\hline & 4.5 & 0.35466 & 0.28538 & 0.29165 & 0.00587 & 0.00504 \\
\hline & 5.0 & 0.32190 & 0.25293 & 0.26231 & 0.00584 & 0.00464 \\
\hline & 5.5 & 0.29258 & 0.22460 & 0.23680 & 0.00570 & 0.00421 \\
\hline \multirow{11}{*}{250} & 0.5 & 0.85018 & 0.83697 & 0.82259 & 0.00032 & 0.00129 \\
\hline & 1.0 & 0.74766 & 0.71873 & 0.70402 & 0.00110 & 0.00260 \\
\hline & 1.5 & 0.66411 & 0.62242 & 0.60949 & 0.00213 & 0.00374 \\
\hline & 2.0 & 0.59343 & 0.54192 & 0.53175 & 0.00318 & 0.00460 \\
\hline & 2.5 & 0.53252 & 0.47373 & 0.46681 & 0.00411 & 0.00515 \\
\hline & 3.0 & 0.47940 & 0.41547 & 0.41201 & 0.00484 & 0.00540 \\
\hline & 3.5 & 0.43269 & 0.36537 & 0.36540 & 0.00537 & 0.00541 \\
\hline & 4.0 & 0.39138 & 0.32207 & 0.32548 & 0.00570 & 0.00524 \\
\hline & 4.5 & 0.35466 & 0.28452 & 0.29110 & 0.00585 & 0.00495 \\
\hline & 5.0 & 0.32190 & 0.25182 & 0.26132 & 0.00585 & 0.00458 \\
\hline & 5.5 & 0.29258 & 0.22328 & 0.23539 & 0.00573 & 0.00419 \\
\hline
\end{tabular}


Table (22): IMSE estimating values of reliability distribution for all methods \& sample size second model for all values of mixed ratio parameter $(\alpha)$.

\begin{tabular}{|c|c|c|c|c|c|c|}
\hline$\alpha$ & $n$ & $T$ & $M$ & $M L E$ & WLS & Better \\
\hline \multirow{6}{*}{0.3} & \multirow[t]{2}{*}{150} & 2 & 4 & 0.003993 & 0.003991 & WLS \\
\hline & & 4 & 8 & 0.00377 & 0.00416 & MLE \\
\hline & \multirow[t]{2}{*}{200} & 2 & 4 & 0.00374 & 0.00362 & WLS \\
\hline & & 4 & 8 & 0.00344 & 0.00378 & MLE \\
\hline & \multirow[t]{2}{*}{250} & 2 & 4 & 0.00363 & 0.00349 & WLS \\
\hline & & 4 & 8 & 0.00338 & 0.00366 & MLE \\
\hline \multirow{6}{*}{0.4} & \multirow[t]{2}{*}{150} & 2 & 4 & 0.00429 & 0.00428 & WLS \\
\hline & & 4 & 8 & 0.00403 & 0.00447 & MLE \\
\hline & \multirow[t]{2}{*}{200} & 2 & 4 & 0.00404 & 0.00393 & WLS \\
\hline & & 4 & 8 & 0.00376 & 0.00409 & MLE \\
\hline & \multirow[t]{2}{*}{250} & 2 & 4 & 0.00395 & 0.00381 & WLS \\
\hline & & 4 & 8 & 0.00369 & 0.00398 & MLE \\
\hline \multirow{6}{*}{0.5} & \multirow[t]{2}{*}{150} & 2 & 4 & 0.00464 & 0.00462 & WLS \\
\hline & & 4 & 8 & 0.00437 & 0.00480 & MLE \\
\hline & \multirow[t]{2}{*}{200} & 2 & 4 & 0.00436 & 0.00424 & WLS \\
\hline & & 4 & 8 & 0.00409 & 0.00443 & MLE \\
\hline & \multirow[t]{2}{*}{250} & 2 & 4 & 0.00426 & 0.00409 & WLS \\
\hline & & 4 & 8 & 0.00401 & 0.00428 & MLE \\
\hline
\end{tabular}

\section{CONCLUSION}

1- The values of estimator of reliability by $M L$ and WLS methods approaches to real values of $R(t)$, at increasing the size of first population especially for model I and III.

2- The values of mean square error for estimated parameter decreases at increasing $\mathrm{T}$ and for the both methods (MLE, WLS).

3- The best estimator for reliability function is obtained at model I and III for all methods, while for model II, there is equality for priority of methods.

\section{SUGGESTION}

1- Generalize this approach for three sub populations, to show the effect of methods of estimation.

2- We can choose another method for estimating parameters and reliability for this mixed Weibull distribution.

3- Using another values of $\mathrm{T}, \mathrm{M}$, to compare the change between results.

4- Applying this study on field work, for comparison. 


\section{REFERENCE}

[1] Al-athari, Faris M. Hassan, Dhwyia S. Ibrahim Nathier A. (2012), "Using Decision Theory Approach to build a model for Bayesian Sampling Plans" American Journal of Mathematics and Statistics. Vol. 2, No.6.

[2] Al - Fawzan, M. (2000), " Methods for estimating the parameters for the weibull distribution", e - mail; mfawzan@ kacst,edu,sa.

[3] Al - Kutbi, H. S. (2005), "On comparison estimation procedure for parameter and survival function exponential distribution using simulation", Baghdad university, collage of Ibn al - Haitham.

[4] Bakar, D., Bridges, D., Hunter, R., Johnson, G., Krupa, J., Murphy, J. and Sorenson, K. (2002)." Gidebook to Decision - Making Methods ", WSRC-IM2002-00002, Department of Energy, USA.

[5] Bather, J. (2000). "Decision Theory". Chichester: John Wiley \& Sons, Inc.

[6] B. Veber, M. Nagode, M. Fajdiga, (2004)"Beyond 'lognormal versus gamma': discrimination among error distributions for generalized linear models [CiTO] Fisheries Research, Vol. 70, No. 2-3. (2004), pp. 351366, doi:10.1016/j.fishres..08.013.

[7] B. Veber, M. Nagode, M. Fajdiga. (2008), "Generalized renewal process for repairable systems based on finite Weibull mixture [CiTO] Reliability Engineering and System Safety, Vol. 93, No. 10. (2008), pp. 1461-1472, doi:10.1016/j.ress.2007.10.003.

[8] C. T. Lin, S. J. S. Wu, N. Balakrishnan, (2004), " Interval estimation of parameters of log-gamma distribution based on progressively censored data" [CiTO] Communications in Statistics-Theory and Methods, Vol. 33, No. 11-12. (2004), pp. 2595-2626.

[9] D. Cousineau, (2009) " Fitting the three-parameter weibull distribution: review and evaluation of existing and new methods" [CiTO] Dielectrics and Electrical Insulation, IEEE Transactions on, Vol. 16, No. 1. pp. 281-288, doi:10.1109/TDEI.2009.4784578.

[10] Dan Ling, Hong-Zhong Huang, Yu Liu, (2009), "A method for parameter estimation of Mixed Weibull distribution [CiTO] In Reliability and Maintainability Symposium, 2009. RAMS 2009. Annual (2009), doi:10.1109/RAMS.2009.4914663.

[11] E. Dick. "Generalized renewal process for repairable systems based on finite Weibull mixture"
(2007) [CiTO] Reliability Engineering and System Safety, Vol. 93, No. 10. (2008), pp. 1461-1472, doi:10.1016/j.ress.2007.10.003.

[12] Gong, Z. (2005),"Estimation of mixed Weibull distribution parameters using SCEN-UA algorithm: Application and comparison with MLE in automotive reliability analysis" Reliability engineering and system safety, pp.1- 8 .

[13] Hassun. Dhwiya. Salman, Ibrahim. Nathier. Abas, Albadri. Faten Farouq. (2012), "Decision Making with applications", Al- jazeera Bureau and publishing (1677). Baghdad. Iraq.

[14] Hassun. Dhwiya. Salman, Ibrahim. Nathier. Abas, Albadri. Faten Farouq. (2012), " Proposed Methods for Estimating Parameters of the Generalized Raylieh Distribution in the Presence of One Outlier ", American Journal of Mathematics and Statistics. Vol. 2, No.6.

[15] K. Krishnamoorthy, Y. Lin, Y. P. Xia, (2009), " Confidence limits and prediction limits for a Weibull distribution based on the generalized variable approach" [CiTO] Journal of Statistical Planning and Inference, Vol. 139, No. 8. (Aug 2009), pp. 2675-2684, doi:10.1016/j.jspi.2008.12.010.

[16] Lawless, J. F. (2003), "Statistical models and methods for life time data", $2^{\text {nd }}$ edition New Jersey, Jhon Wiley, Inc.

[17] L. Pace, A. Salvan, L. Ventura, (2006),"Likelihoodbased discrimination between separate scale and regression models" [CiTO] Journal of Statistical Planning and Inference, Vol. 136, No. 10. (Oct 2006), pp. 3539-3553.

[18] Mosler, K. and Scheicher, C. (2004), "Homogeneity testing in a Weibull mixture model", wiso.fak / wisostatsem / publications / Htiawmm / www.uni.koeln de / wmlx.pdf.

[19] Tsionas, E. G. (2002), "Bayesian analysis of finite mixtures of Weibull distributions", Commu. Stat. theory method, Vol. 31, No. 1, pp. $37-48$.

[20] Z. Yang, M. Xie, A. C. M. Wong, (2007), " A unified confidence interval for reliability-related quantities of two-parameter Weibull distribution" [CiTO]Journal of Statistical Computation and Simulation, Vol. 77, No. 5, pp. 365-378, doi:10.1080/00949650701227452. 